\title{
Polyploid InCidence AND Evolution
}

\author{
Sarah P Otto ${ }^{1}$ and Jeannette Whitton ${ }^{2}$ \\ ${ }^{I}$ Department of Zoology and ${ }^{2}$ Department of Botany, \\ University of British Columbia, Vancouver BC V6T 1Z4 Canada; \\ e-mail: otto@zoology.ubc.ca; jwhitton@interchange.ubc.ca
}

Key Words triploid, tetraploid, animals, plants, gene duplication, rate of evolution

- Abstract Changes in ploidy occurred early in the diversification of some animal and plant lineages and represent an ongoing phenomenon in others. While the prevalence of polyploid lineages indicates that this phenomenon is a common and successful evolutionary transition, whether polyploidization itself has a significant effect on patterns and rates of diversification remains an open question. Here we review evidence for the creative role of polyploidy in evolution. We present new estimates for the incidence of polyploidy in ferns and flowering plants based on a simple model describing transitions between odd and even base chromosome numbers. These new estimates indicate that ploidy changes may represent from 2 to $4 \%$ of speciation events in flowering plants and $7 \%$ in ferns. Speciation via polyploidy is likely to be one of the more predominant modes of sympatric speciation in plants, owing to its potentially broad-scale effects on gene regulation and developmental processes, effects that can produce immediate shifts in morphology, breeding system, and ecological tolerances. Theoretical models support the potential for increased adaptability in polyploid lineages. The evidence suggests that polyploidization can produce shifts in genetic systems and phenotypes that have the potential to result in increased evolutionary diversification, yet conclusive evidence that polyploidy has changed rates and patterns of diversification remains elusive.

\section{CONTENTS}

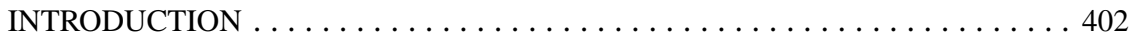

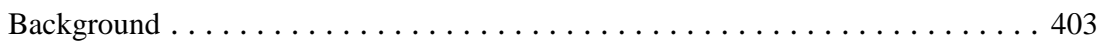

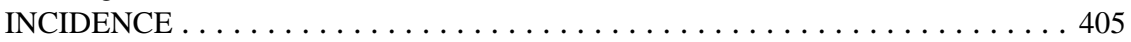

Incidence in Plants . . . . . . . . . . . . . . . . . . . . 405

Incidence in Animals . . . . . . . . . . . . . . . . . . . . . . 409

Phenotypic Effects of Polyploidy . . . . . . . . . . . . . . . . . . 413

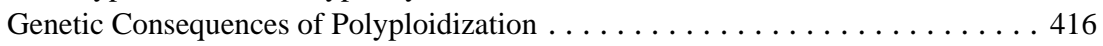

Maintenance of Duplicate Genes and Divergence in Gene Function . . . . . . . . . 425

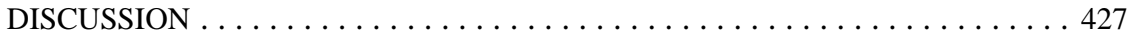

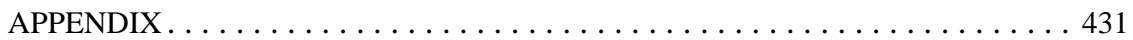




\section{INTRODUCTION}

...polyploidy has contributed little to progressive evolution.

Stebbins (116, p. 132)

...polyploidy, far from playing a secondary role in evolution, has provided the additional, uncommitted gene loci necessary for major steps in the evolution of animals.

Schultz (104)

Polyploidy is widespread in plants, with an estimated frequency between 30 and $80 \%$ (79), and occurs sporadically among animals (69). The evolutionary significance of polyploidy remains a mystery, however. As illustrated by the above quotes, two diametrically opposing views exist, one assigning polyploidy a marginal role in evolution and the other granting it a primary creative role. On the one hand, polyploids may represent a relatively frequent class of mutation, one that occasionally establishes within populations when its phenotypic effects are relatively mild. Simply put, polyploidy may be widespread because it arises repeatedly, without playing a significant role in evolution. Conversely, polyploidy may be common because polyploid species evolve faster or in more novel directions than related diploid species. Under this view, polyploidy promotes adaptive evolutionary change.

Understanding the evolutionary role of polyploidy provides insight into one of the most fundamental questions in evolutionary biology: To what extent is the pace of phenotypic evolution set by the structure and content of the genome? The tempo of evolution may be determined largely by the rate of environmental change, spurred by changes in the abiotic or biotic context in which a species lives, but slowed during periods of stasis (see Reference 67 for an experimental demonstration of the importance of the environment on the rate of evolution in Escherichia coli). Alternatively, the tempo may be set internally and may depend strongly on genomic structure and content. The rate of evolutionary change in a trait not only depends on the strength of selection but also on the form and extent of genetic variability present within a population (39). Thus, evolutionary change could be constrained by the number and type of genes present. If this constraint is substantial, then doubling the number of genes within a genome could have profound long-term effects on genetic variability and on evolution. If, on the other hand, genetic constraints on evolutionary change are minor, polyploidization may make little difference to the tempo of evolution.

In this review, we address the fundamental question of whether polyploidy has played a secondary or an essentially creative role in evolution. We begin by reexamining the incidence of polyploidy in plants and animals. We then review the common phenotypic correlates of polyploids and their immediate selective effects. Third, we address the population genetic consequences of polyploidy on the mutation load and the rate of adaptation. Finally, we review recent genomic studies that shed light on past polyploidization events and their influence on evolution. Although our conclusions are by necessity tentative, evidence suggests that 
the tempo and mode of evolution may have been extensively influenced by the occurrence of polyploidy.

Throughout this paper, we adopt the terminology and conventions employed by Ramsey \& Schemske (99). We use $x$ to denote the base chromosome number of a lineage and $2 n$ to refer to the chromosome number in somatic tissue, regardless of whether an individual is diploid $(2 n=2 x)$, triploid $(2 n=3 x)$, etc. Thus, $n$ represents the gametic or haploid chromosome number expected after meiosis in a sexually reproducing organism. We use the term allopolyploidy to refer to polyploids of hybrid origin, consistent with its first use by Kihara \& Ono (59). By contrast, autopolyploids are formed by the coming together of entire genomes from within a species. It is often assumed that polyploids that form bivalents during meiosis (i.e. exhibit disomic segregation) are allopolyploids, whereas those that form multivalents during meiosis are autopolyploids. These rules are often broken, however (99). In particular, polyploids with tetrasomic segregation (pairing of four homologous chromosomes during meiosis) tend to rediploidize over time as mutations accumulate and chromosomes diverge. Furthermore, autopolyploids in groups with small chromosomes or low chiasma frequencies may exhibit disomic inheritance immediately after their formation (115). Therefore, it is important to keep the mode of origin of polyploidy and the mode of chromosomal segregation distinct.

\section{Background}

Our review aims to uncover the long-term role of polyploidy in evolution. Recent reviews have focused solely on plants and emphasized shorter-term questions. In particular, Ramsey \& Schemske (99) examined the rate of formation of polyploid plants, and Soltis \& Soltis $(110,111)$ reviewed data on the tendency of polyploid taxa to descend from multiple polyploidization events. Here, we briefly review their findings concerning how often and in what manner polyploid populations arise.

Polyploidy represents a special class of mutation and can occur via several routes: genomic doubling, gametic nonreduction, and polyspermy. Genomic doubling occurs in both animals and plants and involves a failure of cell division following mitotic doubling. Similarly, gametic nonreduction involves a failure of cell division during meiosis. Unreduced eggs are a common route to polyploidy in both animals and plants, as is unreduced pollen in plants. Unreduced sperm, however, appear to play a minor role in polyploidization in animals, perhaps because diploid sperm are rarely successful in competition with reduced sperm (see Reference 3 for an exception). Polyspermy is also known in plants and animals and is, for example, the most common mechanism leading to human triploids (119), which comprise $\sim 1-3 \%$ of conceptions (80). Ramsey \& Schemske (99) estimate that autotetraploid plants are formed at a rate on the order of $10^{-5}$ per individual per generation. Allotetraploids are formed at similar rates only if hybrid matings represent at least $2 \%$ of matings in outcrossing groups (99). Recent data 
(reviewed in 118) confirm that autopolyploidy may be a more common phenomenon in plants than had once been thought $(50,114)$.

One of the biggest stumbling blocks to the successful establishment of polyploidy in sexual species is the requirement for a genetically compatible mate. Perhaps because of this obstacle, successful polyploid establishment appears to be facilitated by selfing, asexuality, and perenniality $(12,99,113)$. For example, polyploidy is more often found among perennial plants (113) and insects (71), probably because having a long life span increases the chances that rare events will occur (e.g. polyploidization following hybridization), and allows for mating between polyploids and their offspring. In predominantly outcrossing taxa, an obstacle facing newly formed tetraploids is that they often mate with diploid relatives, producing triploids. Triploidy has generally been thought to be an evolutionary dead-end, because triploids have very low fertility and tend to produce aneuploid gametes, owing to problems of chromosomal pairing and segregation during meiosis. Nevertheless, triploids do produce euploid (haploid, diploid, or triploid) gametes at a low rate $(99,104)$. These euploid gametes can then lead to the production of triploid and tetraploid offspring. Consequently, triploids may actually facilitate the transition from diploidy to tetraploidy by allowing the propagation of polyploid lineages, albeit at a low rate $(54 \mathrm{a}, 99)$.

A potentially important observation is that the rate of polyploid formation in plants and animals varies greatly with environmental circumstances [especially temperature $(15,35,99)]$, parental genotype $(13,99)$, and parental origin [being substantially higher in hybrids than nonhybrids $(54 a, 62,99)]$. This rate variation may be critical to the successful establishment of polyploids in that, occasionally, circumstances may conspire to produce several polyploids within the same generation. A sudden freeze during egg development, the drift to high frequency of a strain tending to produce unreduced gametes, or an unusually high rate of hybrid formation (e.g. in hybrid zones) may generate multiple polyploids that, if there is any tendency towards reproductive isolation from the diploid progenitor, may successfully establish a new polyploid lineage.

The formation of a polyploid lineage from multiple individuals has important ramifications. Genetic variation would be drastically reduced within polyploids formed from a single ancestor, reducing the ability of a polyploid lineage to adapt to its environment and to keep apace of its genetically diverse diploid progenitor. Interestingly, recent studies have suggested that polyploid populations more often have multiple origins than single origins. The plant literature on multiple polyploid origins is reviewed in $(110,111)$. In Table 1 (available at www.annualreviews.org Supplementary Materials), we review the incidence of polyploidy in insects and vertebrates; nine of these polyploid taxa are thought to have multiple origins compared with one considered to have arisen via a single origin. Other invertebrate polyploids with multiple origins are discussed in $(25,33)$. Multiple genetic origins can be explained either by a high rate of polyploidization during the initial establishment of the lineage (as suggested by the previous paragraph) and/or by on-going gene flow with related diploids. In either case, genetic diversity within 
the newly formed polyploid population will be substantially affected. Indeed, studies examining genetic diversity have found that genetic diversity in polyploids is often similar to or higher than related diploids $(19,63,110)$.

\section{INCIDENCE}

\section{Incidence in Plants}

Many familiar crop species, including wheat, maize, sugar cane, coffee, cotton, and tobacco, are polyploid, either through intentional hybridization and selective breeding (e.g. some blueberry cultivars) or as a result of a more ancient polyploidization event [e.g. maize $\sim 11$ MYA (44)]. Polyploidy is also thought to be the mechanism by which certain species have evolved particularly high chromosome numbers, including the stonecrop, Sedum suaveolens [with $2 n=640 \approx 80 x$, the highest known chromosome number among angiosperms (120)] and the fern Ophioglossum pycnostichum [with $2 n=1260 \approx 84 x$, the highest among plants (73)].

Detecting polyploidization events can be enormously challenging. Commonly, polyploidy is inferred when chromosome numbers among closely related species follow a polyploid series (e.g. $2 n=16,32,64)$. Less often, polyploidy is demonstrated by the observation of multivalents during meiosis. Both of these methods, however, are better able to detect recent polyploidization events than ancient ones. Ancient polyploidization events are especially difficult to detect because time erases the signals of duplication; disomic segregation reestablishes, rearrangements scramble chromosomal synteny, and differentiation (or loss) obscures gene copies. Soltis \& Soltis (110) have coined this problem the "paradox of highly diploidized polyploid populations." Over the past few years, extensive genomic analyses have confirmed polyploidization events in ancestors to maize (2), yeast (131), and Xenopus (57). Even genomic analyses can be ambiguous, however. For example, Ohno's (87) hypothesis that two rounds of genome duplication occurred early in the evolution of vertebrates has received both supporting $(4,47,98)$ and contradictory $(77,109)$ evidence from comparative genomic analyses.

To assess the evolutionary significance of polyploidization, it is critical to move beyond identifying particular cases and towards estimating the overall incidence of polyploidy. Previous estimates for the extent of polyploidy in angiosperms vary widely: $20-40 \%$ (113), 57\% (49), and 70\% (48, 79). These estimates measure different quantities, and each suffers from a large degree of extrapolation. Stebbins' (113) estimate was based on the percentage of polyploid species within a genus that had "haploid numbers that were multiples or near multiples of the lowest one found in the genus," which estimates the fraction of species with polyploidy in their history, within the time frame of generic evolution. Grant's (49) estimate was based on the assumption that the ancestral number of chromosomes in angiosperms was 7-9 and that any flowering plant with $n \geq 14$ chromosomes had undergone 
polyploidization at some point during angiosperm evolution. Goldblatt (48) and Masterson (79) argued that Grant's threshold was too conservative and that $\mathrm{n} \geq 11$ was more appropriate, from which they inferred that $70 \%$ of angiosperm species are polyploid. These methods are crude, however, and can only tell us whether polyploidy has occurred at some point in the past. Groups that have polyploidized once and those that have done so repeatedly are treated equally. Using Stebbins' method, for example, a polyploid index of $80 \%$ would be inferred both for a genus containing one species with $n=12$ and four species with $n=24$ and also for a genus containing five species with haploid chromosome numbers $n=12,24,48$, 96 , and 192, respectively; clearly, however, more polyploidization has occurred within the second group. Grant's threshold method would also estimate the degree of polyploidy as $80 \%$ in both of these genera, whereas Goldblatt's and Masterson's threshold method would identify $100 \%$ of these species as polyploids. In addition, the threshold methods suffer from the fact that chromosome numbers will have increased above the threshold in some nonpolyploid taxa by chromosomal fission and will have decreased below the threshold in some polyploid taxa by chromosomal loss and fusion [e.g. Zea mays, which has $n=10$ but is known to be tetraploid (82)]. Estimating the fraction of species that have undergone genome duplication at some point in their evolutionary history has its value, but it provides no clear insight into whether polyploidization has been a rare or frequent event during evolution.

Here we develop a new method to estimate the incidence of polyploidy, which utilizes the distribution of haploid chromosome numbers (Figure 1). A remarkable feature of this distribution is the large excess of even over odd haploid numbers. This saw-toothed pattern is difficult to explain by any mechanism other than frequent polyploidization. This signature of polyploidy arises because the haploid number is always even following genomic doubling, either within a species (autotetraploidy) or following hybridization between species with the same number of chromosomes (monobasic allotetraploidy). Other processes, including chromosome fission, fusion, gain, or loss, tend instead to move species between the categories of even and odd chromosome numbers (Figure 2). Indeed, we can use this saw-toothed signature to provide a simple index of the incidence of polyploidy. If $\rho$ is the rate of polyploidization per unit time, and $\chi$ is the rate by which haploid chromosome numbers increase or decrease by one ("dysploidy"), then the fraction of species $(f)$ that have an even haploid chromosome number obeys the differential equation:

$$
\frac{d f}{d t}=-\chi f+(\rho+\chi)(1-f) .
$$

Over time, the fraction of species with an even $n$ will approach the steady-state value, $f=(\rho+\chi) /(\rho+2 \chi)$. Consequently, the fraction of all karyotypic changes that involve genomic doubling can be estimated by:

$$
P I=\frac{\rho}{\rho+\chi}=\frac{f-(1-f)}{f}=\frac{\# \text { evens }- \text { \# odds }}{\# \text { evens }},
$$




\section{\# Fern Species}

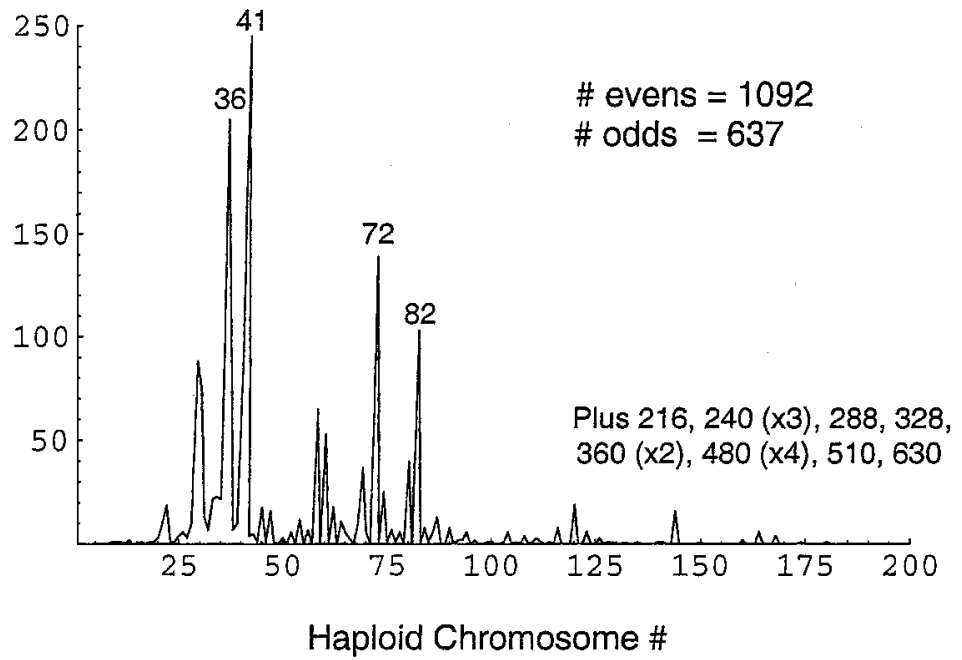

Figure 1 Haploid chromosome numbers in ferns. The distribution of haploid chromosome numbers in Pteridophyta (Filicopsida) was compiled from Löve et al (73) by J Tzenova. Multiple chromosome numbers within species were included separately; circa or unreliable counts were excluded; noninteger haploid numbers (e.g. triploids) were also excluded.

which we call the polyploid index. The polyploid index will be most accurate when there is a low degree of phylogenetic inertia in chromosome number and when long evolutionary time frames are considered. Misleading estimates will be obtained when many species share a particular haploid chromosome number because they are closely related and few karyotypic changes have occurred. To guard against this problem, we calculate the polyploid index only for large taxonomic groups that have a broad array of haploid chromosome numbers with the most common chromosome number (the modal number) represented by at most $15 \%$ of species. Results for ferns, monocots, dicots, crustacean decapods, cockroaches, and mammals are provided in Table 2. Under the null hypothesis that polyploidization does not occur, one can determine the significance of the polyploid index from the binomial distribution with an expectation of $50 \%$ even and 50\% odd chromosome numbers $(P I=0)$. This calculation assumes, however, that the data points are independent, whereas many sibling species share a common chromosome number. Consequently, the number of independent data points will be fewer than the number of species. To address this issue, we calculate an index of support, which indicates the fraction of the data that must be independent for the polyploid index to remain significantly different from zero. When this fraction is low, there is strong support for a non-zero polyploid index. 
Figure 2 A model describing transitions among even and odd chromosome numbers. The polyploid index $(P I)$ is based on the steady-state expectation of this model.

\section{Rate parameters:}

$\chi=\#$ changes by one

$\rho=\#$ doubles

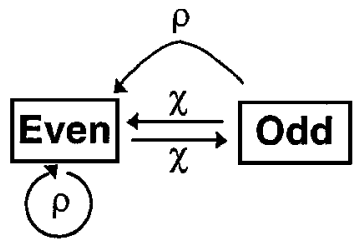

The ferns are well known for having a high degree of polyploidy (127). The ancestral or basal chromosome number within ferns is disputed but is thought to lie within the range of 7 to 13 (127). Using the threshold method to estimate levels of polyploidy (i.e. the proportion of taxa with $n \geq 14$ ), $99.7 \%$ of fern species listed in Löve et al (73) have polyploidy in their history. Given that most ferns have high chromosome numbers relative to angiosperms, the relative importance of polyploidy early in the evolution of ferns ("paleopolyploidy") versus more recent events ("neopolyploidy") has been debated (127). If polyploidization has been rare after the initial radiation of ferns, we would expect a low polyploid index. In

TABLE 2 Polyploid indices $(P I)$ for various groups of plants and animals

\begin{tabular}{|c|c|c|c|c|c|c|}
\hline Taxa & \# Species $^{\mathbf{a}}$ & \# Even & Mode $^{b}$ & $P I^{c}$ & $p$ [support $^{\mathrm{d}}$ & Reference \\
\hline Ferns & 1729 & 1092 & $14.2 \%[41]$ & $41.7 \%$ & $10^{-28}[3.9 \%]$ & 73 \\
\hline Monocots & 4988 & 2963 & $12 \%[7]$ & $31.7 \%$ & $10^{-40}[2.5 \%]$ & 50 \\
\hline Dicots & 11742 & 6441 & $9.8 \%$ [9] & $17.7 \%$ & $10^{-25}[3.7 \%]$ & 50 \\
\hline Herbaceous & 5287 & 3043 & $11.3 \%[8,9]$ & $26.3 \%$ & $10^{-28}[3.5 \%]$ & 52 \\
\hline Woody & 2665 & 1318 & $21.5 \%[13]$ & $-2.2 \%$ & 0.586 & 52 \\
\hline $\begin{array}{l}\text { Decapod } \\
\text { crustaceans }\end{array}$ & 38 & 24 & $10.5 \%[53]$ & $41.7 \%$ & 0.143 & 130 \\
\hline Cockroaches & 107 & 52 & $14.0 \%[19]$ & $-5.8 \%$ & 0.847 & 27 \\
\hline Mammals & 924 & 442 & $8.9 \%$ [19] & $-9.0 \%$ & 0.188 & 130 \\
\hline
\end{tabular}

${ }^{a}$ Number of species with even and odd haploid chromosome numbers were obtained from tables or figures in the references (see Figure 1 for information on ferns).

${ }^{b}$ Mode describes the percentage of species counts with the modal chromosome number (indicated in brackets).

${ }^{\mathrm{c}}$ The $P I$ index is based on equation (2).

${ }^{\mathrm{d}} p$ is the exact binomial probability of getting even:odd counts as far or farther from 1:1 as observed (two-tailed test). The index of support is the percentage of the data that is required to maintain a significant result $(p<0.05)$, which indicates how sensitive a result is to the assumption that chromosome counts are independent. 
contrast, ferns have a high polyploid index (41.7\%), meaning that nearly half of recent changes in haploid chromosome number have occurred via polyploidization. To calibrate this measure per speciation event, we estimated the fraction of speciation events in ferns associated with a change in chromosome number, by totaling the minimum number of chromosome changes found within each genus, i.e. $\sum_{\text {genera }}$ (\# unique $n$ values per genus minus one), and dividing this by the total number of speciation events represented, i.e. $\sum_{\text {genera }}$ (\#species per genus minus one). We ignored genera with a chromosome count from only one species. This procedure indicated that $\approx 16 \%$ of recent speciation events in ferns involved a change in chromosome number. Because chromosome numbers are known from only $\approx 15 \%$ of fern species (73), this estimate is necessarily coarse. Furthermore, there will generally be more chromosome changes and speciation events in the history of these taxa than estimated above. Keeping in mind our uncertainty, we estimate that $\sim 7 \%(=41.7 \% \times 16 \%)$ of speciation events involve polyploidization in ferns. We conclude that polyploidy is an active and on-going process in ferns and one that is involved in a substantial fraction of speciation events.

Polyploidy is also considered to be an important on-going process among angiosperms $(48,49,79,113)$. Consistent with this view, the polyploid index is significantly greater than zero in both monocots and dicots. Interestingly, there is no evidence that recent chromosomal changes in woody dicots involve polyploidization. Instead, the high polyploid index of dicots is entirely due to a high rate of polyploidization among herbaceous dicots. Why there is such a low rate of polyploidization in woody angiosperms is not well understood, but one possibility is that the proper formation of wood fibers by the vascular cambium may constrain cell size changes and hence constrain changes in ploidy level among woody species (113). Similar developmental constraints may explain the exceedingly low representation of polyploidy among gymnosperms (31). To estimate the rate of polyploidization per speciation event in angiosperms, we again calculated the fraction of speciation events associated with a change in chromosome number. We used data from plant families starting with the letters A, F, L, R, and $\mathrm{X}$ in Federov (36), focusing only on genera whose chromosome numbers were summarized in table format. To be conservative, we excluded species that were polymorphic for chromosome number. There were at least 987 chromosomal shifts in 8884 speciation events, implying that the rate of change of chromosome number is $\sim 11 \%$ per speciation event. Multiplying this by the polyploid index, we estimate that $\sim 2-4 \%$ of speciation events in angiosperms involve polyploidization.

\section{Incidence in Animals}

Polyploidy is far rarer in animals than in plants, yet there are hundreds of examples of recent and ancient polyploidization events throughout the animal kingdom. Table 1 (see www.annualreviews.org/Supplementary Materials) lists known polyploid insects and vertebrates, along with their mode of reproduction [summarized in Table 3; for reviews of polyploidy in other invertebrates, see $(12,21,33,65$, 
TABLE 3 Incidence of polyploid species of insects and vertebrates ${ }^{\mathrm{a}}$

\begin{tabular}{llllll}
\hline Taxa & Reproductive mode & $\mathbf{2 x} / \mathbf{3} \boldsymbol{3}$ & $\mathbf{3}$ & $\mathbf{4 x}$ & Other \\
\hline Insects & Parthenogens & 13 & 34 & 16 & 16 \\
& Sexuals & 0 & {$[1]$} & 0 & {$[2]$} \\
\multirow{4}{*}{ Fish } & Parthenogens & 1 & 6 & 0 & 2 \\
& Sexuals & 0 & 0 & $10[1]$ & 12 \\
\multirow{4}{*}{ Amphibians } & $?$ & 0 & 2 & $12[1]$ & 3 \\
& Parthenogens & 1 & 0 & 0 & 2 \\
\multirow{4}{*}{ Reptiles } & Sexuals & 0 & 0 & $18[2]$ & 6 \\
& $?$ & 0 & 0 & 0 & 1 \\
\multirow{2}{*}{ Mammals } & Parthenogens & 3 & $11[1]$ & 0 & 0 \\
& Sexuals & 0 & 0 & 0 & 1 \\
\hline
\end{tabular}

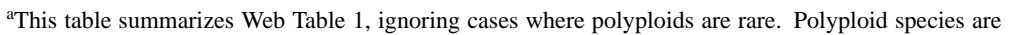
grouped by ploidy level and mode of reproduction, where '?' denotes that the reproductive mode is unknown to the authors. Values in brackets refer to additional cases where there is doubt about the polyploid nature of the species.

101, 122)]. Support for polyploidization varies from karyotypic analyses of closely related taxa [e.g. in red viscacha rats (43)] to genomic sequence analysis (e.g. the putative genome duplications early in the evolution of tetrapods $(4,98)]$.

We calculated the polyploid index for three animal groups (Table 2), and none exhibited a polyploid index significantly different than zero. Decapod crustaceans have a wide range of chromosome numbers, $n=27-188$ (65), and a high polyploid index (41.7\%), but few chromosome counts were available for analysis. The high value of the index is consistent with karyotypic comparisons, suggesting polyploidy in several species of lobsters, crayfish, spiny lobsters, and hermit crabs [in Astacidea, Scyllaridea, and Anomura (65)]. More chromosome data are necessary to improve the power of the analysis and to assess the frequency of polyploidization in Decapoda. In contrast, the polyploid indices of mammals and cockroaches (Blattaria) were slightly negative (i.e. $n$ was more often odd than even), but these values were not significantly different from zero despite the inclusion of $>100$ species. Given that polyploidy is known to be rare in both groups, these analyses serve as a negative control, confirming that the polyploid index is approximately zero when polyploidy has played a relatively minor role in the on-going evolution of a group.

Müller was the first to explain the relative paucity of polyploidy in animals based on the idea that polyploidization would interfere with sex determination in animals (83). Dioecy, where male and female reproductive organs are found in separate individuals, is rare in plants [comprising $\sim 6 \%$ of taxa (100)] but common in animals, where it is often genetically based (20). As noted by Müller, whenever sex is determined by the ratio of $\mathrm{X}$-chromosomes to autosomes (as in Drosophila), newly arisen polyploid species will produce offspring with 
unbalanced ratios leading to disturbed sexual development and sterility. Evidence suggests, however, that sex determination is more often based on the presence or absence of a Y chromosome (88). With a dominant-Y mechanism for sex determination (or equivalently, a dominant-W mechanism in WZ/ZZ species), polyploidy is less likely to disrupt sexual development, but other obstacles arise. In a newly formed population of tetraploids, for example, the sex ratio will be strongly biased towards the heterogametic sex. With male heterogamety, for example, the $\mathrm{Y}$ will have an initial frequency of 50\%, causing males (XYYY, XXYY, XXXY) to be common and females (XXXX) to be rare. This problem will be short-lived, however, because selection for an even sex ratio is strong and rapid (9,39). A second obstacle to the establishment of polyploidy in dioecious taxa is the disruption of dosage compensation whenever there are degenerate sex chromosomes that have evolved mechanisms to balance the ratio of $\mathrm{X}$ to autosomal gene products (88).

These considerations generate the predictions that polyploidy should be more common in animal taxa $(a)$ with asexual or hermaphroditic reproduction, $(b)$ with sex determination based on the presence of a Y chromosome rather than the $\mathrm{X}$ to autosomal ratio, and $(c)$ with non-degenerate sex chromosomes and an absence of dosage compensation. Indeed, polyploidy is highly associated with parthenogenetic reproduction in animals (Tables 1 and 3), even though parthenogenesis is rare (12). Furthermore, among those amphibia and reptiles where polyploidy does occur in sexual groups, heteromorphic sex chromosomes are rare (14), supporting Orr's (88) contention that sex chromosome degeneration may prevent successful polyploidization. Nevertheless, organisms may circumvent potential obstacles to the establishment of polyploidy in unexpected ways. For example, although there is genetic sex determination in diploid Xenopus (WZ/ZZ system), artificially formed hybrids often show temperature-dependent sex determination (63). This flexibility in the sex determination mechanism may allow the continued production of both sexes following polyploidization. Such flexibility may have contributed to the successful establishment of dioecious polyploid animals (Table 1), which are particularly common among amphibia.

Although sex determination and dosage compensation may be important obstacles to the success of polyploidy in animals, additional factors must be at work. Studies investigating asexual species or species lacking chromosomal sex determination find that polyploidy is "surprisingly uncommon" $(21,33,130, \mathrm{p}$. 181). An additional contributing factor to the rarity of polyploids in animals may be that interspecific hybridization is less common, reducing the frequency of allopolyploids (33). It is not clear whether and to what extent this generalization is true, however; certainly hybridization is not uncommon in several animal taxa [e.g. 3130 hybrid species combinations are known among fish (104)]. Indeed, the opposite problem also contributes to the rarity of polyploid animals: hybridization may be too common between newly formed polyploids and their diploid progenitors (130, p. 456). Shifts in flowering time and ecological habitat are frequently found among plants of differing ploidy level (118), which would increase the mating isolation between diploids and polyploids. In contrast, animal mobility would 
reduce the chance that newly formed polyploids are spatially isolated. Interestingly, it has been argued that polyploidy may be found more often among anurans because increased cell volume can affect vocalization, which is a major determinant of reproductive compatibility, thus reducing the extent of polyploid-diploid hybridization (14).

Finally, developmental constraints severely restrict the appearance of polyploids in at least some animal taxa $(114$, p. $368 ; 126)$. Extensive data from chickens and humans suggest that a generalized disruption of development may explain the near absence of polyploid birds and mammals. ZZW chickens that do hatch have an intersex phenotype (1), which is consistent with a Z:autosomal sexdetermination mechanism in birds (26) and with Müller's (83) explanation for the lack of polyploidy in animals. However, mortality is high even among ZZZ individuals, which develop as males (26). In a study of 4182 chicken embryos, haploids (1.4\%), triploids (0.8\%, $9 \mathrm{ZZZ}, 7 \mathrm{ZZW}, 15 \mathrm{ZWW})$, and tetraploids $(0.1 \%$, 1 ZZZZ, 1 ZZWW) were found, none of which survived to hatching (13). Disruption of sex determination is unlikely to explain the fact that all of these genotypes die early in development. Furthermore, dosage compensation is not known to occur in birds (103), which argues against Orr's (88) explanation for the lack of polyploid birds. Indeed, the fact that both ZZZZ and ZZWW tetraploids die as embryos bolsters the claim that polyploids suffer from a general disruption of development rather than a particular problem associated with having chromosomal sex determination. Similar observations apply to human polyploids. Approximately $5 \%$ of natural abortuses are polyploid (28). Although most triploids and tetraploids have severe defects and fail to survive to term, livebirths do occasionally occur with infants surviving for up to two years $(55,107)$. Imprinting has been implicated as a possible mechanism causing developmental abnormalities in triploids $(32,80)$, based on the observation that survival is higher for digynic triploids (formed from polyploidization in the egg) than for dispermic triploids (formed from polyploidization in the sperm or, more commonly, fertilization by two sperm). Consistent with what is known about imprinting in mammals, digynic triploids develop at an appropriate rate but display stunted growth, while dispermic triploids are larger than expected based on their developmental stage (80). Tetraploid newborns, both XXXX [female (107)] and XXYY (male (72)], have been reported and are thought to result from a failure of cell division in the zygote. Although polyploidy can disrupt normal sexual development and cause abnormal genital formation in humans (102), the fact that polyploid survival is much lower than that of trisomics involving the sex chromosomes (XYY, XXY, and XXX) and is associated with much more severe abnormalities and early mortality regardless of genotype suggest that a general disruption of development and not a disruption of sexual development, per se, explains the absence of polyploidy in adult humans. One factor contributing to the high rate of natural abortion of polyploid humans is abnormal placental growth $(28,45)$, perhaps related to changes in cell size or to a disruption of the balance between maternally and paternally imprinted genes. 
Although the above obstacles undoubtedly contribute to the rarity of polyploidy in animals, they are not absolute. Polyploid races or species are found sporadically in many groups of animals (Table 1). These species result from the occasional congruence of factors lessening the developmental and ecological constraints on polyploid formation. Nevertheless, these polyploid species may represent nothing more than evolutionary dead-ends. If this were true, however, we would expect very few animal groups to be anciently polyploid. Although it is difficult to confirm ancient polyploid events, several examples are known (Table 1). Among fish, larger taxonomic groups of polyploids include the Actynopterygii (the highly speciose ray-finned fishes), Catostomidae, Salmonidae, the subfamily Schizothoracinae (Cyprinidae), the monophyletic group CorydorasAspidoras-Brochis (Callichthyidae), the subgenus Barbus (Cyprinidae), and the subgenus Labeobarbus (Cyprinidae). Among amphibia, polyploid groups include the Sirenidae and Xenopus (Pipidae). In addition, vertebrate genomes contain extensive collinearity in genetic content on different chromosomes $(47,98)$, supporting Ohno's (87) contention that vertebrates underwent two genomic doubling events early in their evolution. These ancient polyploids indicate that polyploid groups can flourish over evolutionary time, disproving the claim (e.g. 116) that the rate of evolution will be so slowed when multiple homologous chromosomes are present.

\section{Phenotypic Effects of Polyploidy}

The role of polyploidization as a creative force in producing evolutionary novelty will be mediated through its effects on the phenotype. Therefore the fundamental question that must be addressed is whether polyploidization, in and of itself, tends to produce phenotypic shifts that have adaptive potential. Levin (68) considered the phenotypic effects of polyploidy in flowering plants and their role in producing evolutionary novelty. He concluded that there was ample evidence from flowering plants that "chromosome doubling may propel a population into a new adaptive sphere" and "bring about abrupt, transgressive, and conspicuous changes in the adaptive gestalt of populations within microevolutionary time." Here we briefly summarize and update his presentation, reaching a similar, if still tentative, conclusion.

Isolating the direct effects of genome doubling using naturally occurring polyploids is complicated by the difficulty of identifying populations that differ only in ploidy. The effects of traits like hybridity and asexual or vegetative reproduction that frequently occur in polyploid lineages can be confounded with the effects of polyploidy. For example, in allopolyploids, the direct effects of polyploidy will be difficult to separate from the effects of hybridity. While comparisons with diploid hybrids could help tease apart these effects, in practice, suitable populations may not exist. In addition, as noted by Levin (68) among others, it is difficult to isolate the immediate effects of polyploidization from changes that have accumulated subsequent to changes in ploidy. 
Effects on Cell Size and Growth Rates Among the most common and universal effects of polyploidization is increased cell volume (24), although the extent to which this occurs varies (114) and may depend on environmental conditions (B Mable, personal communication). Indeed, stomatal cell size in plants is frequently used as an indirect measure of ploidy level (e.g. 79). Likewise, the diameter of pollen grains, comprising 2-3 nuclei in flowering plants, is also used to infer ploidy. In addition, there is evidence from some species of amphibia (11) and plants (23) that over time, the cell volume of polyploids tends to decrease towards the levels of related diploids. Changes in cell volume result in changes in surface to volume ratios of cells, which may alter the rate of metabolic processes, especially those that involve membranes $(24,68,129)$. These sorts of metabolic changes are likely at the root of changes in growth rates that are frequently detected in polyploid lineages. Developmental rates have been shown to be altered in polyploid plants, amphibia, and insects, with polyploids generally having slower developmental rates than related diploids (e.g. 35, 73a, 125). On the other hand, polyploid plants frequently have larger seeds than related diploids, and this increase can lead instead to higher rates of early development $(123,125)$. Such changes in developmental rates may affect the likelihood of establishment of seedlings in resource-limited environments and may result in niche differentiation as a byproduct of polyploidization.

Effects on Size and Shape Given the changes in cell shape and metabolic and developmental processes described above, effects on overall size and shape of organs and whole organisms would be predicted. Although these are frequently found, many polyploids are described as falling within the range of morphological variation of their diploid progenitors, with only subtle phenotypic effects (e.g. 16, 22, 85). In plants, polyploidy is sometimes, but not always, associated with larger overall size (114). In vertebrates, polyploidy tends to have little to no effect on body size $(35,76,104)$, but it tends to increase size in invertebrates (12, pp. 266, 300-301).

Polyploidization often has some effect on organ structure and function, but such effects tend to be highly idiosyncratic (114). Bretagnolle \& Lumaret (17) attempted to isolate the direct effects of polyploidization in triploids and tetraploids experimentally produced from the diploid plant, Dactylis glomerata subsp. lusitanica. Although diploids and polyploids did not differ in overall biomass, polyploids were found to have broader, thicker leaves, fewer stems per plant and fewer inflorescences, increased seed weight, but fewer seeds overall. In hylid frogs, vocalization differs between the diploid Hyla chrysoscelis and the related tetraploid $H$. versicolor (81) but is very similar in the diploid and tetraploid leaf-frogs, Phyllomedusa distincta (53).

Effects on Reproductive Systems Among the most common shifts in reproductive system that correlate with the occurrence of polyploidy is asexual reproduction. While most apomictic plants are polyploid, most polyploid plants are not apomictic. In animals, however, approximately two thirds of polyploids reproduce 
parthenogenetically (Table 1, Table 3). Interestingly, this fraction is not consistent across taxa. The majority of polyploid insects and reptiles reproduce parthenogenetically, but the majority of polyploid fish and amphibia reproduce sexually. Although the association between parthenogenesis and polyploidy is common to both plants and animals, the reasons for the association may be quite different (117). In animals, parthenogenesis tends to arise in diploids; thereafter, matings between parthenogenetic diploids and related sexuals frequently generate polyploids through the union of unreduced eggs and sperm. In contrast, in flowering plants, polyploidization often appears to predate apomixis (117). For example, recent studies indicate that the genes for apomixis can only be transmitted in unreduced gametes $(86,96)$.

In addition to shifts to asexual reproduction, other changes in breeding systems have been noted in plants. For example, genetic self-incompatibility systems may break down in polyploids, resulting in higher selfing rates in polyploids than in their diploid progenitors (114, pp. 306-307; 128a). Further, polyploidization may alter floral traits, including the relative sizes and spatial relations of floral organs (18). These changes may shift biotic interactions with pollinators, which may lead to further selection for divergence in reproductive traits.

Geographical Distribution and Ecological Correlates Polyploids are frequently said to have broader ecological tolerances than their diploid progenitors $(68,71$, 106, 121). Among the explanations for this observation is the idea that increased heterozygosity can provide metabolic flexibility to cope with a broader array of conditions. For example, multimeric enzymes assembled from two or more subunits will have more different forms of the enzyme available in polyploid heterozygotes, each of which may be most effective under slightly different conditions. Another possibility is that the polyploid species that successfully establish are a biased subset of all polyploids that arise. A particularly strong bias may be that polyploids that are able to persist are more likely to inhabit different niches than their diploid progenitors. Niche separation reduces competition and reduces the chance of cross-ploidy matings, which tend to produce low fitness progeny (e.g. triploids). Consequently, niche partitioning may be highly selectively favored in newly established polyploids $(78,122)$.

In what may be a related phenomenon, polyploid plants and animals frequently occur at higher altitudes and more polar latitudes than their diploid progenitors $(6,7,10,12,34,56,70)$. While numerous taxa display these trends, no satisfactory general explanation has been proposed, and exceptions have been noted (114). Because cold treatments can increase the frequency of unreduced gamete formation $(15,35,99)$, and these are involved in most polyploidization events, it is tantalizing to hypothesize that this relationship may reflect different rates of origination of polyploids in colder climates. However, we do not know the historical distributions of most taxa, and therefore, in most cases, we do not know if polyploids originated in these colder climes, or whether they expanded their ranges into these regions owing to their broader tolerances. It has also been proposed that larger cell size 
may be at the root of the selective advantage that some polyploids appear to display in these areas (34).

\section{Genetic Consequences of Polyploidization}

...the large amount of gene duplication dilutes the effects of new

mutations and gene combinations to such an extent that polyploids have great difficulty evolving truly new adaptive gene complexes.

Stebbins (116, p. 133).

It is often argued that the evolutionary success of polyploids is hampered by the inefficiency of selection when multiple alleles are present at each gene. Indeed, the spread of a favorable allele from a given frequency is slower at higher ploidy levels (132, p. 50), because the selective effects of an allele are partially off-set by the presence of alternate alleles. Populations of varying ploidy level are not, however, expected to start from the same allele frequency, and this difference must be taken into account in determining the evolutionary effects of polyploidy. In particular, because polyploid individuals carry more alleles, each individual has a greater chance of carrying a new beneficial mutation (93). Similarly, alleles that have recently become beneficial will tend to be at higher frequency in polyploid populations if they were previously deleterious, because deleterious alleles persist for longer and reach higher frequencies when masked. Surprisingly, there has been relatively little work on the basic population genetic consequences of polyploidy. Here we explore the mutation load and rate of adaptation of polyploid species. Although these evolutionary factors may affect the persistence of a newly formed polyploid lineage, the ecological effects of polyploidization are more likely to determine establishment in the short-term. Nevertheless, the genetic load and rate of adaptation may have longer-term effects on the persistence and diversification of polyploids once formed. As we shall see, polyploid populations can have a transiently lower deleterious mutation load than populations of lower ploidy level, and they can, under certain circumstances, adapt at a faster rate.

Notation In the following sections, we consider the dynamics at one locus in a population of size $N$ and of ploidy level $c$. Matings between individuals of different ploidy levels are not considered, nor are ecological interactions. The locus is subject to mutations at rate $\mu$ (if deleterious) or $v$ (if beneficial) per generation. Let allele $A$ be wild type with a relative fitness of 1 in haploids, and let allele $a$ be mutant with fitness $1 \pm s$, depending on whether beneficial ( + ) or deleterious (-) mutations are considered. We will assume that the fitnesses of $A, A A$, and $A A A A$ individuals are equal, as are the fitnesses of $a, a a$, and $a a a a$ individuals. This symmetry ensures that there are no intrinsic fitness advantages of one ploidy level over another and allows us to explore the effects of masking alone. In diploids, heterozygotes have fitness $1 \pm h s$, where $h$ measures the dominance of the mutant allele. In tetraploids, there are three heterozygous types, $A A A a$, 
$A A a a$, and $A a a a$, whose fitnesses are defined as $1 \pm h_{1} s, 1 \pm h_{2} s$, and $1 \pm h_{3} s$, respectively. To simplify notation, we shall write $\sigma_{\mathrm{c}}$ for the fitness effects of a single mutation in an organism of ploidy level $c$. That is, $\sigma_{1}=s, \sigma_{2}=h s$, and $\sigma_{4}=h_{1} s$, which are all assumed to be non-zero (completely recessive alleles are not treated).

When considering polyploids with multisomic inheritance, we assume, for simplicity, that the gene of interest is located near the centromere. Hence, we ignore the phenomenon of double reduction, where recombination between the centromere and a gene allows an $A_{1} A_{2} A_{3} A_{4}$ tetraploid to produce $A_{1} A_{1}$ gametes, for example (30). Double reduction tends to make selection in tetrasomic tetraploids more similar to that in diploids, because the double-diploid tetraploids ( $A A A A$, $A A a a, a a a a$ ) become more common relative to the $A A A a$ and Aaaa genotypes. We also assume that selection is sufficiently weak that the population will be in nearly Hardy-Weinberg proportions (132).

Masking Deleterious Mutations Increasing ploidy can provide a selective advantage by masking the deleterious fitness effects of mutations (54, p. 110). Previous models have shown that the selective advantage of diploid life cycles over haploid life cycles is greatest when deleterious mutations are recessive or nearly so $(64,91,95)$. Similarly, we might expect polyploids to have a fitness advantage over lower ploidy levels through masking. The situation is complicated, however, by the fact that individuals of higher ploidy have a higher chance of bearing a mutation and the fact that deleterious mutations persist for longer in populations of higher ploidy levels [as shown by (91) in a comparison of diploid and haploid life cycles]. Assuming a large population size, low deleterious mutation rate $(\mu)$, and tetrasomic inheritance in tetraploids, the equilibrium frequency of the mutant allele $(q)$ becomes $\mu / \sigma_{\mathrm{c}}$ in both asexual and randomly mating sexual populations. This frequency increases with ploidy level for deleterious mutations that are partially recessive and masked (i.e. $h<1 / 2$ and $h_{l}<1 / 2 h$ ), which is consistent with data from diploids (108). At equilibrium, the amount by which mean fitness is reduced by the presence of deleterious mutations (the mutation load) is the frequency of heterozygous individuals $\left(\approx c \mu / \sigma_{\mathrm{c}}\right)$ times their average selective cost $\left(\sigma_{\mathrm{c}}\right)$. Therefore, the mutation load is approximately $c \mu$ and rises linearly with ploidy level, $c$.

For a newly produced tetraploid population, however, these equilibrium calculations are inappropriate. If there were multiple origins of the tetraploid population, then the initial mutant allele frequency in the tetraploid population is expected to equal $\mu / \sigma_{2}$, i.e. the diploid mutant allele frequency at equilibrium. Newly formed tetraploid populations would then have a mutation load equal to $4 \mu \sigma_{4} / \sigma_{2}=$ $4 \mu h_{1} / h$. This load will be lower than the equilibrium load of the diploid progenitor population whenever $h_{l}<h / 2$, i.e. whenever $A A A a$ tetraploids are nearer in fitness to $A A$ diploids than $A a$ diploids (the equivalent of being partially recessive). Essentially, there is an immediate advantage to polyploidization whenever individuals of higher ploidy levels are better able to mask single-copy 


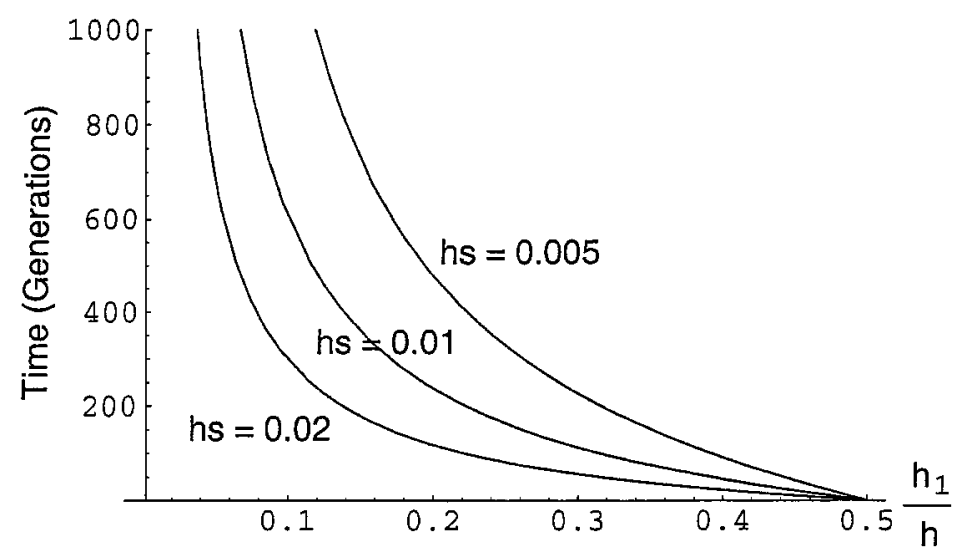

Figure 3 The time required for the mutation load in a newly formed tetraploid population to rise above the load of a diploid population. Assuming that mutations are rare and that selection is relatively weak, this time equals $\log _{\mathrm{e}}[2(1-f)] /(f h s)$, where $f=h_{l} / h$. For tetraploid populations to have a reduced genetic load initially, $f$ must be less than $1 / 2$, consistent with deleterious alleles being partially recessive. Here, $h s$ is set to $0.005,0.01$, and 0.02 (the scale on the y-axis is inversely proportional to $h s$ ).

deleterious mutations than individuals of lower ploidy levels. This advantage is transient, however, because deleterious alleles increase in frequency over time until $A A A a$ genotypess are relatively common. In a newly formed tetraploid population, mean fitness decreases exponentially towards its equilibrium level at rate $\sigma_{4}$. The number of generations that a tetraploid population is expected to have a lower genetic load than its diploid ancestor is shown in Figure 3, which shows that the transient advantage of a lower load may persist for many generations, especially when masking is strong. The picture is complicated if the tetraploid population starts from a single individual, but the qualitative outcome is similar when averaged over the genome. As long as a single copy of a deleterious mutation is more masked in a tetraploid than in a diploid, a newly formed tetraploid population will have, on average, a lower genetic load initially. This advantage dissipates over time, with tetraploid populations ultimately having a higher genetic load.

Before leaving the topic of deleterious mutations, it is worth discussing another possible advantage to sexual tetraploids: deleterious mutations are masked in the gametophyte or gamete phase. When selection acts on the gametophyte (as in many plants), a new tetraploid population may gain an immediate advantage due to masking deleterious mutations in the diploid phase. Again, this advantage is transitory, because such deleterious alleles will eventually rise to a higher frequency in tetraploid populations as a side-consequence of their being masked. Nevertheless, the initial success of a sexual tetraploid population may be enhanced by the additional protection derived from having two copies of every gene in the gametophyte. 
Altered Rates of Adaptation In polyploids, any benefit imparted by a new advantageous mutation is diluted by the effects of alternative alleles at the same locus. As noted by Paquin \& Adams (93), however, organisms with a higher ploidy level have a higher chance of bearing new beneficial alleles. In the final balance, whether polyploids evolve more or less rapidly depends on several key factors, including their mode of reproduction, population size, and dominance coefficients.

Asexuals Because polyploidy often occurs in asexual lineages especially in animals, we begin by discussing the rate of evolution in asexual populations. The spread of beneficial mutations is hampered in asexual populations by the fact that beneficial mutations occurring in separate individuals cannot be recombined into the same genome (39). Because only one asexual lineage will be the ultimate ancestor of the descendant population, the rate of evolution of an asexual population is limited to those beneficial mutations that appear in this lineage. In the Appendix, we estimate the effect of ploidy on the rate of increase of fitness owing to the accumulation of beneficial mutations, which arise within a population at rate $c N v$. For an additively acting allele (with $\sigma_{c}=\sigma_{l} / c$ ), the rate of fitness increase is always highest for haploids $(c=1)$. More generally, we note that the rate of fitness increase lies within the ranges:

$$
h^{2} \lesssim \frac{\text { Rate of increase of fitness in diploids }}{\text { Rate of increase of fitness in haploids }} \lesssim 2 h^{2}
$$

and

$$
\frac{h_{1}^{2}}{h^{2}} \lesssim \frac{\text { Rate of increase of fitness in tetraploids }}{\text { Rate of increase of fitness in diploids }} \lesssim 2 \frac{h_{1}^{2}}{h^{2}} \text {. }
$$

The rates approach the lower limits of these ranges in large populations $(N v \gg 1)$ and approach the upper limits in small populations where few beneficial mutations appear each generation $(N v \ll 1)$.

Because it is reasonable to assume that $h_{l}<h<1$, the lower limits of Equations 3 indicate that haploids will adapt fastest, followed by diploids, and finally tetraploids in large asexual populations with high rates of beneficial mutations $(N v \gg 1)$. Under these circumstances, the rate of adaptation is not limited by the appearance of beneficial mutations but by their rate of spread to high frequency, which is always faster in haploids (132, p. 50). For smaller populations, however, the rate at which beneficial mutations appear and avoid loss, while rare, is low enough to limit the rate of adaptation. Because more beneficial mutations appear in polyploid populations, the rate of adaptive evolution can increase with ploidy level. The upper limits of Equation 3 predict that, in small populations $(N v \ll 1)$, diploids will adapt faster than haploids when $h>0.71$ and that tetraploids will adapt even faster if $h_{l}>0.71 \mathrm{~h}$. Unfortunately, we have very few data on the dominance coefficient of newly arisen beneficial alleles in diploids, let alone in tetraploids. Nevertheless, we might expect the fitness benefits of an allele to 


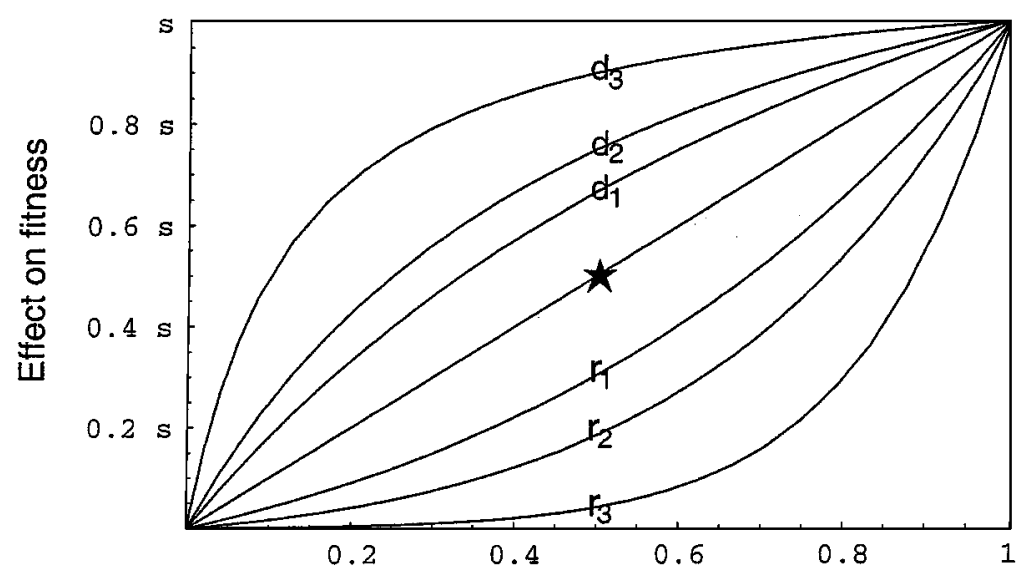

Fraction of alleles of the novel type

Figure 4 Relationship between allele dosage and fitness effect. The fraction of alleles within an individual that are of the new beneficial type $a$ is drawn on the x-axis. Wild-type individuals have an x-value of zero, individuals carrying only $a$ alleles have an x-value of one, and intermediate genotypes have intermediate values (e.g. $1 / 2$ for $A a$ or AAaa individuals and $1 / 4$ for $A A A a$ individuals). The $y$-axis is the selection coefficient acting on individuals of the given allele dosage. Hypothetical curves are drawn based on an additive model (straight line, denoted by a star), on a hyperbolic model (curves denoted by $d_{1}-d_{3}$, which exhibit dominance), and on an exponential model (curves denoted by $r_{1}-r_{3}$, which exhibit recessivity). The hyperbolic model is expected based on the relationship between flux and enzyme activity in metabolic control theory (58).

scale with the dose of that allele according to functions of the shapes illustrated in Figure 4 [the hyperbolic curves $d_{l}-d_{3}$ are based on the effects of varying the activity of an enzyme in a metabolic pathway; akin to Figure 4 in (58)]. Interestingly, for the types of curves shown, diploids never have the highest rate of fitness increase according to Equation A2 (see appendix). When dominance is high enough (e.g. curves $d_{2}$ and $d_{3}$ ), fitness is expected to increase fastest in tetraploid populations, otherwise it will increase fastest in haploid populations. In summary, asexual populations of higher ploidy level can adapt faster than those of lower ploidy levels if the population is relatively small and if beneficial alleles tend to be partially dominant.

Sexuals In sexual populations with ample genetic mixing, beneficial mutations that occur in separate individuals can be brought together by recombination (39). Indeed, the fixation probability of a mutation is only slightly affected by the dynamics at other loci unless linkage is tight (8). Consequently, the fixation probability of each beneficial mutation is very nearly $2 \sigma_{c}$ (assuming directional selection on an allele that is not fully recessive). Therefore, mutations that successfully become established appear within a population at a rate of $2 \sigma_{c} c N v$ per generation. 
Multiplying this rate by the resulting increase in fitness once the mutant allele is fixed, we estimate that the rate of increase in fitness is $\Delta \mathrm{W}_{1 \mathrm{n}}=(2 s v N) s$ in haploids, $\Delta \mathrm{W}_{2 \mathrm{n}}=(4 h s v N) s$ in diploids, and $\Delta \mathrm{W}_{4 \mathrm{n} \text {-tetrasomic }}=\left(8 h_{1} s v N\right) s$ in tetraploids with tetrasomic inheritance. Therefore, sexual tetrasomic tetraploids will adapt faster than diploids whenever $h_{l}>h / 2$. For tetraploids with disomic segregation, however, new beneficial alleles can spread to fixation only at one of the two gene copies (unless gene conversion occurs). If the fitness of such AAaa individuals equals $1+h_{2} s$, then the rate of increase in fitness will equal $\Delta \mathrm{W}_{4 \mathrm{n} \text {-disomic }}=\left(8 h_{1} s v N\right) h_{2} s$. Now, $h_{1} h_{2}$ must be greater than $h / 2$ for tetraploids with disomic inheritance to evolve faster than diploids. Figure 5 summarizes these results. It is necessary, however, to determine the biologically relevant part of this parameter space. Once again, we use the relationships between allele dosage and fitness illustrated in Figure 4, which suggest that parameters near the dotted curve may be most common. Along this curve, either haploids have the highest average rate of fitness increase (for beneficial alleles that are partially recessive) or tetraploids do (for beneficial alleles that are partially dominant). There is no justification for the widespread notion that evolution proceeds fastest in diploids.

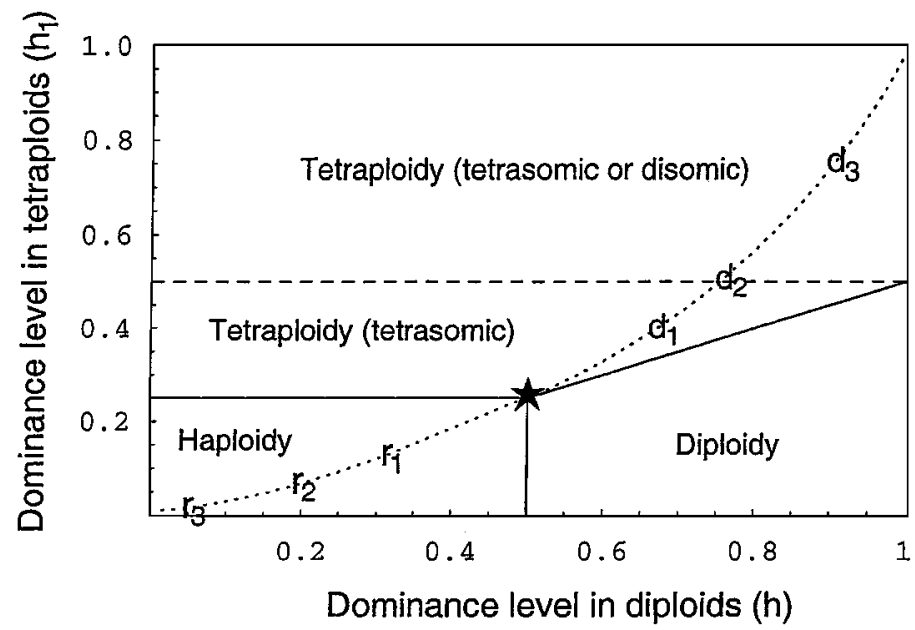

Figure 5 Dominant expression of new beneficial alleles favors higher ploidy levels. In sexual populations, the rate of adaptation will be highest for the ploidy level indicated on the graph, all else being equal. The $\mathrm{x}$-axis and $\mathrm{y}$-axis correspond to the dominance level of a single-copy beneficial allele in diploids and tetraploids, respectively (i.e. $h$ and $h_{l}$ ). For tetraploids with disomic segregation, the fitness of $A a$ and $A A a a$ individuals are set equal ( $h=h_{2}$ ), in which case tetraploids evolve faster than diploids in the region above the dashed line. A star is placed at the point where an additively acting allele would lie. The dotted curve corresponds to the dominance relationships expected based on curves of the form illustrated in Figure 4. 
The above discussion is based, however, on the assumption that newly arisen mutations are the basis of adaptive change. It may be that beneficial alleles are previously deleterious alleles whose selection coefficients have become positive in response to environmental changes. Because deleterious mutations tend to be more masked and more frequent in populations of higher ploidy level, polyploids may be better poised to respond rapidly to changes in the environment. As we shall show, whether polyploids adapt faster under these circumstances depends critically on the population size. We consider a population at mutationselection balance, with deleterious alleles at frequency $q_{c d}=\mu / \sigma_{c d}$, where the subscript $d$ emphasizes that $\sigma_{c d}$ refers to past selection against the allele while it was deleterious. If the number of copies $\left(c N q_{c d}\right)$ of the previously deleterious allele is small, such that the probability that at least one of them fixes is much less than one (i.e. for populations of small to moderate size; $N \mu \ll 1$ ), then the increase in fitness based on the fixation of beneficial alleles that were previously held at a mutation-selection balance is approximately $2 \sigma_{c}\left(c N q_{d}\right) s$ for haploids, diploids, and polyploids with multisomic segregation. As long as selection for the allele when beneficial is not much weaker than it was when deleterious $\left(\sigma_{c} / \sigma_{c d}>1 / c\right)$, increasing ploidy will result in a faster rate of adaptation. The advantage of polyploidy will be most pronounced when the allele is absent or in few copies in diploid populations but tends to be found in multiple copies in polyploid populations (i.e. $2 N q_{2 d}<1$ for diploids but $4 N q_{4 d} \gg 1$ for tetraploids), which can occur if deleterious alleles are well masked and if the population size is moderately small. In this case, the newly beneficial allele can rise immediately in frequency in the polyploid population, whereas haploid and diploid populations must wait for the next appearance of the allele by mutation.

If, on the other hand, the population is so large that enough copies of the previously deleterious allele are present to guarantee that at least one will fix, then the rate of adaptation is no longer set by the probability of carrying a beneficial allele but rather the rate of spread of these alleles. In this case, haploid populations nearly always adapt faster than diploid populations, which adapt faster than polyploid populations, because of the decreasing efficiency of selection with ploidy level (132).

Overall The blanket statement that polyploids adapt more slowly than diploids is false. In small to moderately sized populations, the rate of fitness increase depends less on the efficiency of selection and more on how often mutations appear and establish within populations. Consequently, the rate of adaptation can be faster for higher ploidy levels as long as beneficial alleles are partially dominant. This basic conclusion holds for sexual and asexual polyploids, whether adaptive change results from novel mutations or previously deleterious alleles.

The above calculations are predicated on several simplifying assumptions, which were chosen to focus our attention on the costs and benefits of changing ploidy level alone. In particular, we assumed that $(a)$ the populations being 
compared were of equal size, $(b)$ the selective advantage or disadvantage of an allele was the same regardless of ploidy level if it was the only allele present (i.e. $s$ was treated as constant), and $(c)$ the deleterious $(\mu)$ and beneficial $(v)$ mutation rates were constant per allele. As with any two populations, however, these parameters are likely to differ between a polyploid population and a related diploid population. For example, polyploid populations sometimes inhabit marginal habitats with relatively low population densities (suggesting a lower $N$ ), although polyploids occasionally have much wider ranges (and a higher $N$ ) than related diploid species (122). Furthermore, polyploids are generally not morphologically or physiologically identical to diploids, so the selective advantages and disadvantages of mutant alleles in polyploids need not be related to these coefficients in diploids. For example, immediate changes in gene expression occur upon polyploidization in yeast (42). Some of these changes appear to regulate cell cycle progression (and hence cell size), cell shape, cell clumping, and fungal invasiveness. Metaphorically, a newly produced polyploid population may occupy a different position on the adaptive landscape (132), and the spectrum of fitness effects of alleles may be greatly altered.

An intriguing possibility is that the rate of beneficial mutations may differ among ploidy levels. In particular, if survival and reproduction depend on the proper expression of a particular allele, any mutation that changes its function or pattern of expression will be deleterious in haploids, but some of these mutant alleles may be beneficial in diploids or tetraploids as long as the essential allele is still present and active. Similarly, if the proper expression of an allele within gametes is essential, mutations to this allele may be strongly deleterious in diploids but potentially advantageous in tetraploids. Such phenomena would lead the beneficial mutation rate $(v)$ to increase with ploidy level. Of course, the ultimate outcome of such selection also depends on the ploidy level and mode of segregation. In diploids and tetrasomic tetraploids, a balanced polymorphism will result, where neither the newly functioning allele nor the old essential allele is stably inherited. In disomic tetraploids, on the other hand, the fixation of both alleles on different pairs of chromosomes is possible, allowing their stable inheritance. Indeed, changes in gene function or in the timing and tissue specificity of gene expression have been found between duplicate genes in yeast (131), catostomid fish (38), and Xenopus (57). The possibility exists that genomic duplication released constraints on selection and allowed evolution to proceed in a novel direction, which was, perhaps, prohibited in the absence of gene copies (131).

Altered Linkage Arrangements The evolutionary consequences of polyploidy discussed above result from a change in allelic copy number, which alters both the chance of mutation and the strength of selection on any particular allele. Polyploidization has additional genetic effects that may influence the success of polyploid lineages. With an increasing number of chromosomes and disomic segregation, the average rate of recombination between loci rises. Thus, Grant (51, 


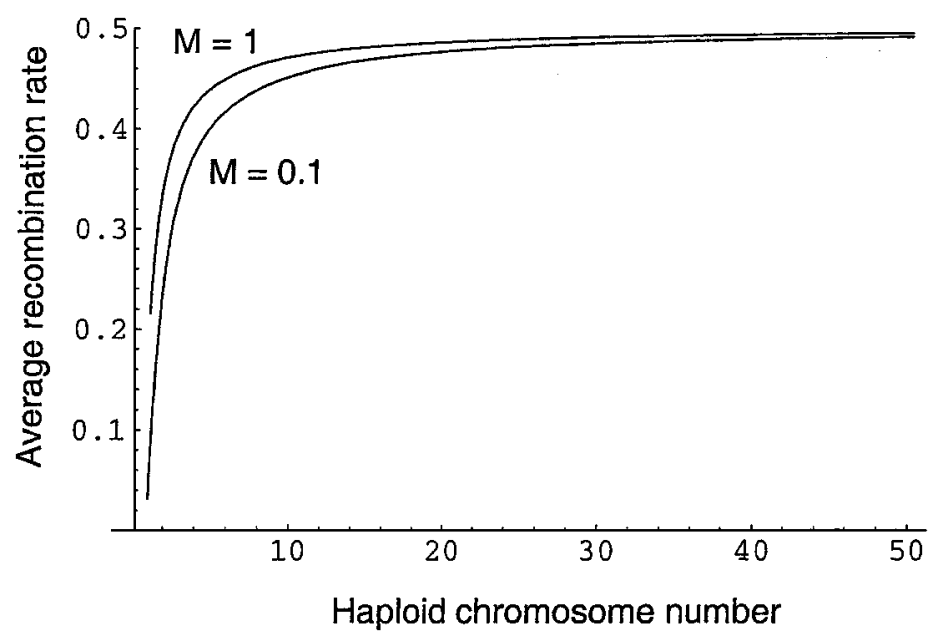

Figure 6 Effect of a change in chromosome number on the rate of recombination between an average pair of loci. This graph assumes that there are $n$ chromosomes (with disomic inheritance) of approximately equal length, $M$, measured in Morgans. The average rate of recombination is $1 / 2$ times the chance that the loci are on different chromosomes plus the average rate of recombination between loci on the same chromosome, calculated using Haldane's mapping function and multiplied by the chance that the two loci are on the same chromosome.

p. 394) argued that larger chromosome numbers would be "favored by selection for open recombination systems." This effect is likely to be weak, however, in any organism with more than a handful of chromosomes (Figure 6). With more than five chromosomes ( $\geq 0.1$ Morgan in length), doubling the number of chromosomes will at most increase the average rate of recombination between randomly chosen pairs of loci by $10 \%$. While the effect on the average level of recombination is modest, selection for increased recombination between certain pairs of loci may favor polyploids. That is, linked loci in a diploid may become unlinked in a polyploid if silencing occurs at opposite genes on the duplicate chromosomes. If the two loci are routinely under directional selection for novel function, the rate of adaptation may be increased by this physical separation $(8,90)$. Recombination is not always advantageous (37), however, and the increase in recombination may reduce polyploid fitness, especially if co-adapted gene complexes are dispersed.

Gene expression and regulation may also be affected by changes in the genomic context in which genes occur following polyploidization. Evidence is mounting that genomic repatterning in polyploids is often extensive and can be rapid $(66,111)$. As a striking example, Song et al (112) created polyploid Brassica hybrids and observed extensive genomic rearrangements within five generations. One possible explanation for such rapid changes is that transposable elements that 
are silent in one parental line become active in polyploids of hybrid origin (129a). Such transposable elements may contribute to physical changes in the karyotype (e.g. translocations, fusions, fissions) and may hasten gene silencing of duplicate gene copies. Such massive and rapid changes in genome structure must often be deleterious, but occasional advantageous variants might arise. Certainly, genomic rearrangements would augment the genetic variation observed in a new polyploid population. Although genomic repatterning may often contribute to the rapid extinction of polyploid lineages, the rare successful lineages may be especially likely to diverge enough in ecological requirements to coexist with or displace their diploid progenitors.

\section{Maintenance of Duplicate Genes and Divergence in Gene Function}

...duplications enable genes to make evolutionary experiments which have previously been forbidden, liberating them from incessant natural selection whose overwhelming activity is eliminating variants.

Kimura (60, p. 16)

The ultimate contribution of polyploid events to evolution would be minor if the fate of most duplicate genes is to be silenced. Early work on duplicate gene evolution predicted just such an outcome (e.g. 40, 54; see 84 for additional references). According to this classical view, partially recessive deleterious mutations would rise in frequency with little opposition from selection as long as a second functional gene was present. Once a deleterious allele fixed in one gene, however, selection would act more strongly to preserve function of the duplicate gene, making the next deleterious mutation more likely to fix in the first gene as well. Over time, mutational decay would eventually silence one member of most gene pairs. Only in those very rare instances where a beneficial mutation that causes divergence in function between duplicate genes arises before the decay process has proceeded too far would both gene copies be preserved (e.g. 54, 87, 128). Walsh (128), for example, estimated that about $99 \%$ of duplicate genes would devolve into pseudogenes by this process.

Data from a variety of ancient polyploids suggest, however, that a much larger fraction of duplicate gene copies is retained over much longer periods of evolutionary time than predicted. The fraction of genes retained in duplicate has been estimated as $\sim 8 \%$ in yeast over $\sim 100 \mathrm{MY}(105), \sim 72 \%$ in maize over $\sim 11 \mathrm{MY}$ $(2,44), \sim 77 \%$ in Xenopus over $\sim 30$ MY $(57), \sim 70 \%$ in salmonids over $25-100$ MY (5), $\sim 47 \%$ in catastomids over $\sim 50 \mathrm{MY}$ (38), and $\sim 33 \%$ in vertebrates over $\sim 500 \mathrm{MY}$ (84). Such high levels of maintenance have led evolutionary biologists to reassess the selective forces acting on duplicate genes, with implications for the role of polyploidy in evolution.

The maintenance of duplicate genes is rendered much more likely than in the classical model if (a) deleterious mutations affect fitness even when present in a 
single copy, $(b)$ there is selection for increased gene expression, and/or $(c)$ gene copies rapidly diverge in function. The first explanation is particularly likely for genes encoding multidomain proteins, which interact with several other proteins, because point mutations tend to disrupt protein-protein interactions and have a partially dominant effect on fitness (46). Consistent with this explanation, many of the genes maintained in duplicate in Xenopus are involved in protein-protein interactions (57). Support for the second explanation comes from yeast, where highly expressed genes (measured by mRNA levels), including heat shock, glucose metabolic, and cytosolic ribosomal genes, were more likely to be retained in duplicate than genes with low levels of expression (105). Nevertheless, many duplicate genes with low expression levels in yeast have also been retained, and Seoighe \& Wolfe (105) suggest that, in these cases, divergence in function has played a role in maintaining duplicate genes. This suggestion is confirmed by the observation that $\sim 10 \%$ of the genes retained in duplicate belong to slightly different functional categories in the Yeast Proteome Database (105). This third explanation, divergence in gene function, is especially likely when degenerative mutations can occur in different functional or regulatory domains of duplicate genes $(41,74)$. In this model, deleterious mutations accumulate in each gene copy disrupting some but not all functions of the gene, until both genes become essential for some of the subfunctions of the original single-copy gene. This phenomenon, termed sub-functionalization (41), ensures that both gene copies remain under selection and allows their preservation over longer periods of evolutionary time $(41,74)$. Not only does this increase the probability that adaptive mutations occur before gene silencing, but it also eases pleiotropic constraints. Selection on a single gene with multiple functions is constrained to favor the fittest sequence averaged over all of the roles of the gene; following duplication and sub-functionalization, mutations that are beneficial to only a subset of the roles of a gene may be favored and spread in the gene copy that eventually takes over these roles (74). The fact that duplicate genes often differ in the timing of expression and/or the pattern of expression in different tissues is consistent with the sub-functionalization model $(38,41,57)$. More direct support comes from examples where the shared expression pattern of duplicate genes coincides with the total expression pattern of single-copy orthologues in related organisms (41).

Under each of these scenarios, duplicate genes maintained over time are not expected to be released from selection at the amino acid level. This prediction contrasts with that of the classical model, which assumes that selection in both copies is initially relaxed. Data examining the level of selective constraints in Xenopus suggest that both duplicate genes are generally subject to purifying selection (57), with synonymous substitutions occurring more frequently than nonsynonymous changes for all 17 pairs of genes examined. Consequently, duplicate genes in polyploid lineages are often preserved in function by purifying selection for long enough to allow for beneficial mutations to arise in each and, hence, for their diversification over time. 


\section{DISCUSSION}

For greater changes the chance of improvement diminishes progressively, becoming zero, or at least negligible, for changes of a sufficiently pronounced character.

Fisher (39)

Based on a compelling geometrical argument, Fisher (39) noted that large mutations would almost surely reduce fitness, either aiming the phenotype away from a fitness peak or causing it to overshoot it. On the basis of Fisher's argument, modern biologists give little credence to the role of "hopeful monsters" in evolution. Consequently, it is puzzling that one of the most drastic possible types of mutation, polyploidization, has occurred frequently throughout evolution. Polyploidy is widespread in plants and makes several appearances in animals (69; Tables 1 and 3), which suggests that polyploids are either not monsters or happen to be particularly hopeful monsters.

Although polyploidization would appear to be the most extreme possible genetic change, the phenotypic changes that it causes are often surprisingly subtle and follow few general rules. The fact that polyploids often develop functionally with few inherent effects on phenotype calls into question the assumption that a change in ploidy is a drastic mutation. Because growth necessarily involves the cyclic doubling and halving of the genome during mitosis (and an even further halving during meiosis), not to mention the widespread phenomenon of endopolyploidy, all organisms must have evolved tolerance to changes in ploidy level. In other words, organisms may be preadapted to withstand changes in ploidy levels, offering a possible resolution to the puzzle of how such drastic mutations persist. Nevertheless, the phenotypic changes that polyploidization does produce can be enormously important for the evolutionary success of newly formed polyploid lineages. Changes in features such as metabolism, developmental rates, gene regulation, and physiological tolerances can alter biotic interactions, ecological tolerances, and facets of reproductive isolation such as mating behavior and breeding system. Such traits play critical roles in the establishment and diversification of newly formed polyploid lineages.

The frequency of polyploidy varies but is particularly high in ferns and angiosperms. Prior estimates for the level of polyploidy in these groups assessed not the rate of polyploidization but the extent to which species have a polyploid history, whether recent or ancient. Such estimates provide a poor assessment of the on-going role of polyploidy in evolution. We have developed a new method to estimate the frequency of polyploidy that relies on the fact that polyploidization leads to an excess of even over odd haploid chromosome numbers (Table 2). We estimate that $\sim 2-4 \%$ of speciation events in angiosperms and $\sim 7 \%$ in ferns are associated with polyploidy. Indeed, polyploidization may be the single most common mechanism of sympatric speciation in plants. One reason that polyploidy may be a particularly common speciation mechanism is that it can be accompanied by 
phenotypic changes that allow niche partitioning between newly formed species and their ancestors. Although polyploidy is associated with many fewer speciation events in animals, there are, nevertheless, hundreds of cases of polyploid animals. Furthermore, evidence is accumulating that points to the role of polyploidy early in the evolution of major groups of animals, including vertebrates, ray-finned fish, salmonids, and catostomids.

That polyploidy has occurred repeatedly throughout evolution does not, however, prove that it has played a major creative role in the evolutionary process. Stebbins $(114,116,117)$ argued against the creative role of polyploidy on the basis of several lines of evidence. First, he argued that polyploidy would retard the rate of adaptive evolution because the effects of beneficial alleles would be "diluted" by the expression of duplicate alleles (116, p. 133). Second, he noted that polyploidy is often associated with relatively unspecialized development (117). Third, Stebbins (115, pp. 199-201) noted that most major groups of plants have a range of haploid chromosome numbers with similar lower limits, suggesting that morphological divergence among angiosperm families predated the polyploid events that led to massive increases in chromosome number within the groups. Finally, he argued that animals have been enormously successful in terms of sheer taxonomic diversity, the evolution of specialization, and the evolution of complex morphological features without the aid of frequent polyploidization events (114). Stebbins concludes that "polyploidy, although it multiplies greatly the number of species and sometimes genera present on the earth, retards rather than promotes progressive evolution" (114, p. 369).

While it is important to remain skeptical, there are strong counter-points to each of the above lines of evidence. First, as we have seen, polyploids can adapt faster than diploids, especially if beneficial mutations appear within relatively small populations and have a partially dominant effect on fitness. Second, one can easily turn the argument that polyploids are rarely specialists on its head; the evolutionary significance of polyploidy may indeed lie in the tendency of polyploids to have a broader ecological tolerance (e.g. 114, 117) and more generalist life history. Third, it is extraordinarily difficult to infer ancient polyploidization events, and it may very well be that a common ancestor of all modern plants was itself polyploid. And, finally, the evolutionary "success" of animals may have been intricately connected with early polyploid events, even if the on-going contribution of polyploidization is small.

While there are reasons to doubt Stebbins' sweeping claims against the evolutionary significance of polyploidy, there is very little hard evidence about the influence of polyploidy on the tempo or mode of evolution. The fundamental question is: To what extent does the doubling of gene number increase the rate of morphological evolution or diversification? Certainly, there has been much speculation that polyploidy promotes adaptive evolution:

Because teleosts are the most species-rich group of vertebrates and exhibit tremendous morphological diversity, it is tempting to speculate that the 
duplication event [in ray-finned fish] may have provided gene copies that helped spur the teleost radiation.

Amores et al (4)

It is interesting to speculate that emergence of the complex head structures of the vertebrates has a basis in the amplification of the homeobox clusters.

Pendleton et al (94)

The proposed genome duplication [in yeast] may have been instrumental in its evolutionary adaptation to anaerobic growth; for example, the duplicate genes include several pairs that are regulated differently under aerobic and anaerobic conditions... as well as several genes encoding sugar transporters.

Wolfe \& Shields (131)

Unfortunately, convincing data on this subject remain to be gathered. The definitive test would examine whether increased rates of speciation or morphological evolution follow polyploid events, assessed in a phylogenetic context involving multiple independent transitions to polyploidy. It is not enough to prove, for example, that novel traits appearing over evolutionary time develop from modifications in duplicated genes, as has been suggested (124), because these novel traits may well have evolved in the absence of gene duplication. Indeed, after polyploidization, we would expect evolutionary change to involve gene duplicates, not necessarily because having gene copies was essential for the evolutionary transition, but because selection acts upon the genetic material at hand, which includes the gene copies. Consequently, polyploidization may not have had an impact on the mode or direction of evolution, even if we now observe that gene duplicates function in different ways.

To explore the influence of polyploidy on taxonomic diversity, we determined the relationship between the number of species in each of 200 dicot genera whose level of polyploidy was assessed by Stebbins (113). As shown in Figure 7, there is a small positive relationship between level of polyploidy and species richness $(0.01<\mathrm{p}<0.05)$. Similar results were reported by Petit \& Thompson (97), who examined taxonomic richness in 50 angiosperm genera in the flora of the Pyrenees. In both cases, taxonomic diversity displays a nonlinear relationship with the degree of polyploidy, with genera having a high fraction of polyploids being less speciose. Unfortunately, this pattern is consistent with the explanation that older genera have a greater mixture of ploidy levels and a higher species richness. To get around this problem requires a phylogenetically correct analysis, where, for example, sister genera of equal age are compared in terms of polyploidy and species richness. This analysis is made difficult by several factors: data on chromosome numbers are lacking for many genera, ploidy level is often difficult to assess, and sister taxa often have similar levels of polyploidy. As a final complicating factor, species richness may be higher in clades with a higher level of polyploidy simply because polyploidy is a speciation mechanism. The ideal study would therefore analyze cases where the only polyploid event occurred on the basal branch to one of the sister groups. 


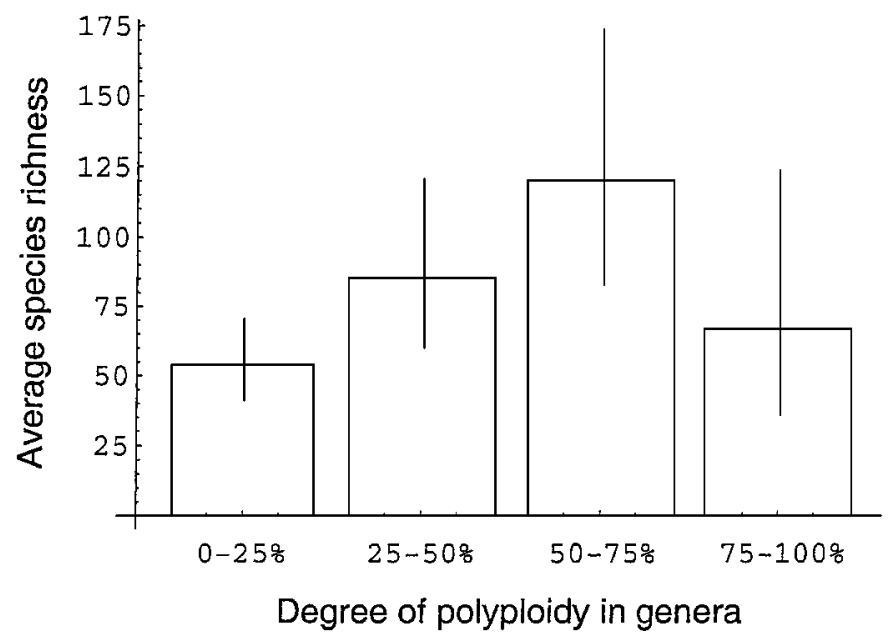

Figure 7 Relationship between ploidy level and species richness in dicots. Genera were classified by Stebbins (113) as 0-25\% (101 genera), 25-50\% (54 genera), 50-75\% (27 genera), and $75-100 \%$ (14 genera) polyploid. The number of species per genus was obtained from Mabberley (75). Species numbers were ln-transformed to satisfy normality assumptions, the means and their $95 \%$ confidence limits were obtained and are illustated (on the original scale of species numbers), and an ANOVA was performed. The ANOVA indicated a significant interaction between ploidy level and taxonomic diversity $(\mathrm{p}=0.017)$. A linear regression based on ranks gave a significantly positive relationship, with the average rank in diversity increasing by 4.3 per ploidy category $(\mathrm{p}=0.017)$. These results were only marginally significant (both $\mathrm{p}=0.11)$ if small genera $(<10$ species $)$ were removed from the analysis.

In the absence of such an ideal analysis, it is difficult to draw firm conclusions about the effects of polyploidy on speciation and extinction rates. Figure 7 and the analysis by Petit \& Thompson (97) are consistent with, but do not prove, that polyploidization has played a positive role in generating evolutionary diversity.

There are theoretical reasons to expect polyploidy to boost rates of adaptive evolution. Polyploids have a greater chance of bearing new beneficial alleles and are better poised to evolve novel functions in duplicated gene families. Yet, the role of polyploidy in evolution remains enigmatic. Phylogenetic analyses promise to clarify the relationship between polyploidy and the tempo of evolutionary change. Genomic analyses promise to reveal more ancient polyploidy events and to determine the specific genetic changes that have followed. These efforts will undoubtedly shed light on the role that polyploidy has played in the major evolutionary transitions of eukaryotes.

\section{ACKNOWLEDGMENTS}

We are enormously grateful to Jordana Tzenova, Maren Friesen, and Kim Ryall, who as student research assistants compiled the chromosome data. We thank Ryan 
Garrett, Barbara Mable, Dolph Schluter, and Michael Whitlock for insightful discussions about the evolutionary role of polyploidy. Finally, we thank Wyatt Anderson, Alex Grant, Jana Heilbuth, Kent Holsinger, and Barbara Mable for providing helpful comments on the manuscript. Support was provided by the Natural Sciences and Engineering Research Council, Canada (to SPO and JW) and the Peter Wall Institute for Advanced Studies (to SPO).

\section{APPENDIX}

We adapt the methods used by Crow \& Kimura (29) and Orr \& Otto (89) to estimate the rate at which fitness increases in a population of ploidy level, $c$. This method estimates the number of generations that must pass, on average, before an individual carrying a beneficial mutation will carry a second beneficial mutation. In a purely apomictic population with $c>1$, a beneficial mutation will spread to fixation on only one of the homologous chromosomes, generating permanent heterozygosity (61). Therefore, we consider only the selective effects of a mutant allele when present in a single-copy, $\sigma_{c}$.

With a constant number of individuals, the chance that a new beneficial mutation survives loss while rare is approximately $2 \sigma_{c}(54)$. Once a beneficial allele becomes established and begins to spread, additional beneficial mutations arising in individuals that do not carry the first mutation are unlikely to fix (if the selection coefficients are similar). When a second beneficial mutation with selective advantage $\sigma_{c}^{\prime}$ arises in the subset of the population carrying the first mutation, it will actually have a higher chance of fixing than $2 \sigma_{c}^{\prime}$, because it occurs within a group of individuals that is growing (because they carry the first beneficial allele). This important effect has been ignored in previous calculations of the rate of adaptive evolution in asexuals $(29,89)$. As the frequency $\left(p_{t}\right)$ of the first beneficial mutation rises logistically over time, $\mathrm{p}_{\mathrm{t}}=\mathrm{p}_{0} /\left(\mathrm{p}_{0}+\left(1-\mathrm{p}_{0}\right) \mathrm{e}^{-\sigma_{c} \mathrm{t}}\right)$, the probability of fixation $\left(P_{t}\right)$ of the second beneficial mutation is:

$$
P_{t} \approx 2 \sigma_{c}^{\prime} \frac{\sigma_{c}^{\prime}+\sigma_{c}}{\sigma_{c}^{\prime}+p_{t} \sigma_{c}}
$$

(92). Consequently, the rate at which beneficial alleles appear that survive loss while rare is $P_{t}$ times the number of individuals carrying the first allele: $p_{t}(c N v)$. The total number of generations $(g)$ that must pass, on average, between the appearance of two successful beneficial mutations can be found by setting $\int_{0}^{g} P_{t} p_{t} c N v d t$ to one and solving for $g$. The expected rate of fixation of adaptive mutations is then the inverse of $g$. Finally, this rate of fixation must be multiplied by $\sigma_{c}$ to get the overall rate at which fitness increases due to the incorporation of new beneficial mutations, giving

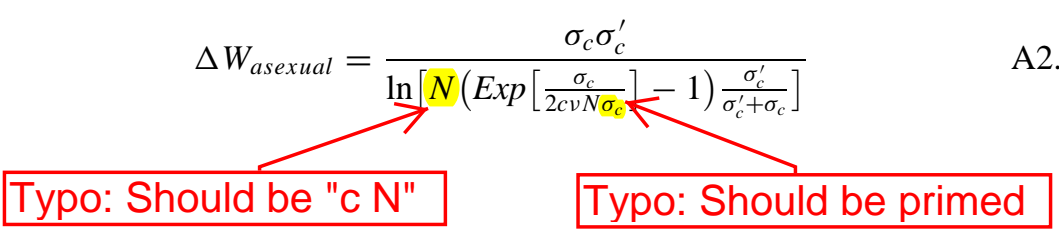


Equation (A2) is used to derive equations (3) in the text, where it is assumed that beneficial alleles have a constant selective advantage $\left(\sigma_{c}^{\prime}=\sigma_{c}\right)$.

\section{Visit the Annual Reviews home page at www.AnnualReviews.org}

\section{LITERATURE CITED}

1. Abdel-Hameed F, Shoffner RN. 1971. Intersexes and sex determination in chickens. Science 172:962-64

2. Ahn S, Tanksley SD. 1993. Comparative linkage maps of the rice and maize genomes. Proc. Natl. Acad. Sci. USA 90:7980-84

3. Alves MJ, Coelho MM, Prospero MI, Collares-Pereira MJ. 1999. Production of fertile unreduced sperm by hybrid males of the Rutilus alburnoides complex (Teleostei, Cyprinidae). An alternative route to genome tetraploidization in unisexuals. Genetics 151:277-83

4. Amores A, Force A, Yan YL, Joly L, Amemiya C, et al. 1998. Zebrafish hox clusters and vertebrate genome evolution. Science 282:1711-14

5. Bailey GS, Poulter RT, Stockwell PA. 1978. Gene duplication in tetraploid fish: model for gene silencing at unlinked duplicated loci. Proc. Natl. Acad. Sci. USA 75:5575-79

6. Barata C, Hontoria F, Amat F, Browne R. 1996. Competition between sexual and parthenogenetic Artemia: temperature and strain effects. J. Exp. Mar. Biol. Ecol. 196:313-28

7. Barata C, Hontoria F, Amat F, Browne R. 1996. Demographic parameters of sexual and parthenogenetic Artemia: temperature and strain effects. J. Exp. Mar. Biol. Ecol. 196:329-40

8. Barton NH. 1995. Linkage and the limits to natural selection. Genetics 140:821-41

9. Basolo AL. 1994. The dynamics of Fisherian sex-ratio evolution: theoretical and experimental investigations. Am. Nat. 144:473-90

10. Beaton MJ, Hebert PDN. 1988. Geo- graphic parthenogenesis and polyploidy in Daphnia pulex Leydig. Am. Nat. 132:83745

11. Beatty RA. 1957. Parthenogenesis and Polyploidy in Mammalian Development. Cambridge: Cambridge Univ. Press

12. Bell G. 1982. The Masterpiece of Nature: The Evolution and Genetics of Sexuality. Berkeley: Univ. Calif. Press

13. Bloom SE. 1972. Chromosome abnormalities in chicken (Gallus domesticus) embryos: types, frequencies and phenotypic effects. Chromosoma 37:309-26

14. Bogart JP. 1980. Evolutionary implications of polyploidy in amphibians and reptiles. See Ref. 69, pp. 341-78

15. Bogart JP, Elinson RP, Licht LE. 1989. Temperature and sperm incorporation in polyploid salamanders. Science 246:103234

16. Borrill M, Linder R. 1971. Diploidtetraploid sympatry in Dactylis (Gramineae). New Phytol. 70:1111-24

17. Bretagnolle F, Lumaret R. 1995. Bilateral polyploidization in Dactylis glomerata L. subsp. lusitanica: occurrence, morphological and genetic characteristics of first polyploids. Euphytica 84:197-207

18. Brochmann C. 1993. Reproductive strategies of diploid and polyploid populations of arctic Draba (Brassicaceae). Plant Syst. Evol. 185:55-83

19. Brochmann C, Soltis DE, Soltis PS. 1992. Electrophoretic relationships and phylogeny of nordic polyploids in Draba (Brassicaceae). Plant Syst. Evol. 182:3570

20. Bull JJ. 1983. Evolution of Sex Determining Mechanisms. Reading, MA: Benjamin/Cummings 
21. Burch JB, Jung Y. 1993. Polyploid chromosome numbers in the Torquis group of the freshwater snail genus Gyraulus (Mollusca: Pulmonata: Planorbidae). Cytologia 58:145-49

22. Burton TL, Husband BC. 1999. Population cytotype structure in the polyploid Galax urceolata (Diapensiaceae). Heredity 82:381-90

23. Butterfass T. 1987. Cell volume ratios of natural and induced tetraploid and diploid flowering plants. Cytologia 52:309-16

24. Cavalier-Smith T. 1978. Nuclear volume control by nucleoskeletal DNA, selection for cell volume and cell growth rate, and the solution of the DNA C-value paradox. J. Cell Sci. 34:247-78

25. Chaplin JA, Hebert PDN. 1997. Cyprinotus incongruens (Ostracoda): an ancient asexual? Mol. Ecol. 6:155-68

26. Clinton M. 1998. Sex determination and gonadal development: a bird's eye view. $J$. Exp. Zool. 281:457-65

27. Cohen S, Roth LM. 1970. Chromosome numbers in Blattaria. Ann. Entomol. Soc. Am. 63:1520-47

28. Creasy MR, Crolla JA, Alberman ED. 1976. A cytogenetic study of human spontaneous abortions using banding techniques. Hum. Genet. 31:177-96

29. Crow JF, Kimura M. 1965. Evolution in sexual and asexual populations. Am. Nat. 99:439-450

30. Crow JF, Kimura M. 1970. An Introduction to Population Genetics Theory. New York: Harper \& Row

31. Delevoryas T. 1980. Polyploidy in gymnosperms. See Ref. 69, pp. 215-18

32. Dietzsch E, Ramsay M, Christianson AL, Henderson BD, de Ravel TJ. 1995. Maternal origin of extra haploid set of chromosomes in third trimester triploid fetuses. Am. J. Med. Genet. 58:360-64

33. Dufresne F, Hebert PDN. 1994. Hybridization and origins of polyploidy. Proc. R. Soc. London Ser. B 258:141-46

34. Dufresne F, Hebert PDN. 1998.
Temperature-related differences in lifehistory characteristics between diploid and polyploid clones of the Daphnia pulex complex. Ecoscience 5:433-37

35. Fankhauser G. 1945. The effects of changes in chromosome number on amphibian development. Q. Rev. Biol. 20:2078

36. Federov AA. 1974. Chromosome Numbers of Flowering Plants. Koenigstein, Germany: Otto Koeltz Sci.

37. Feldman MW, Otto SP, Christiansen FB. 1997. Population genetic perspectives on the evolution of recombination. Annu. Rev. Genet. 30:261-95

38. Ferris SD, Whitt GS. 1979. Evolution of the differential regulation of duplicate genes after polyploidization. J Mol. Evol. 12:267-317

39. Fisher RA. 1930. The Genetical Theory of Natural Selection. Oxford, UK: Oxford Univ. Press

40. Fisher RA. 1935. The sheltering of lethals. Am. Nat. 69:446-55

41. Force A, Lynch M, Pickett FB, Amores A, Yan YL, Postlethwait J. 1999. Preservation of duplicate genes by complementary, degenerative mutations. Genetics 151:153145

42. Galitski T, Saldanha AJ, Styles CA, Lander ES, Fink GR. 1999. Ploidy regulation of gene expression. Science 285:251-54

43. Gallardo MH, Bickham JW, Honeycutt RL, Ojeda RA, Kohler N. 1999. Discovery of tetraploidy in a mammal. Nature 401:341

44. Gaut BS, Doebley JF. 1997. DNA sequence evidence for the segmental allotetraploid origin of maize. Proc. Natl. Acad. Sci. USA 94:6809-14

45. Genest DR, Roberts D, Boyd T, Bieber FR. 1995. Fetoplacental histology as a predictor of karyotype: a controlled study of spontaneous first trimester abortions. Hum. Pathol. 26:201-9

46. Gibson TJ, Spring J. 1998. Genetic redundancy in vertebrates: polyploidy and persistence of genes encoding multidomain 
proteins. Trends Genet. 14:46-49

47. Gibson TJ, Spring J. 2000. Evidence in favour of ancient octaploidy in the vertebrate genome. Biochem. Soc. Trans. 28:259-64

48. Goldblatt P. 1980. Polyploidy in angiosperms: monocotyledons. See Ref. 69, pp. 219-39

49. Grant V. 1963. The Origin of Adaptations. New York: Columbia Univ. Press

50. Grant V. 1971. Plant Speciation. New York: Columbia Univ. Press

51. Grant V. 1982. Chromosome number patterns in primitive angiosperms. Bot. Gaz. 143:390-94

52. Grant V. 1982. Periodicities in the chromosome numbers of the angiosperms. Bot. Gaz. 143:379-89

53. Haddad CFB, Pombal JP Jr, Batistic RF. 1994. Natural hybridization between diploid and tetraploid species of leaffrogs, genus Phyllomedusa (Amphibia). J. Herpetol. 28:425-30

54. Haldane JBS. 1933. The Causes of Evolution. London: Longwood Green

54a. Harlan JR, DeWet JM. 1975. On Ö. Winge and a prayer: the origins of polyploidy. Bot. Rev. 41:361-90

55. Hasegawa T, Harada N, Ikeda K, Ishii T, Hokuto I, et al. 1999. Digynic triploid infant surviving for 46 days. Am. J. Med. Genet. 87:306-10

56. Havel JE, Hebert PDN, Delorme LD. 1990. Genotypic diversity of asexual Ostracoda from a low arctic site. J. Evol. Biol. 3:391-410

57. Hughes MK, Hughes AL. 1993. Evolution of duplicate genes in a tetraploid animal, Xenopus laevis. Mol. Biol. Evol. 10:1360-69

58. Kacser H, Burns JA. 1981. The molecular basis of dominance. Genetics 97:639-66

59. Kihara H, Ono T. 1926. Chromosomenzahlen und systematische Gruppierung der Rumex-Arten. Z. Zellforsch. Mikrosk. Anat. 4:475-81

60. Kimura M. 1983. The Neutral Theory of Molecular Evolution. Cambridge: Cambridge Univ. Press

61. Kirkpatrick M, Jenkins CD. 1989. Genetic segregation and the maintenance of sexual reproduction. Nature 339:300-1

62. Kobel HR. 1996. Allopolyploid speciation. In The Biology of Xenopus, ed. RC Tinsley, Kobel HR, pp. 392-401. Oxford: Oxford Univ. Press

63. Kobel HR, Du Pasquier L. 1986. Genetics of polyploid Xenopus. Trends Genet. 2:310-15

64. Kondrashov AS, Crow JF. 1991. Haploidy or diploidy: Which is better? Nature 351:314-15

65. Lécher P, Defaye D, Noel P. 1995. Chromosomes and nuclear DNA of Crustacea. Invertebr. Reprod. Dev. 27:85-114

66. Leitch IL, Bennett MD. 1997. Polyploidy in angiosperms. Trends Plant Sci. 2:47076

67. Lenski RE, Travisano M. 1994. Dynamics of adaptation and diversification: a 10,000-generation experiment with bacterial populations. Proc. Natl. Acad. Sci. USA 91:6808-14

68. Levin DA. 1983. Polyploidy and novelty in flowering plants. Am. Nat. 122:1-25

69. Lewis WH. 1980. Polyploidy: Biological Relevance. New York: Plenum

70. Little TJ, Hebert PDN. 1997. Clonal diversity in high arctic ostracodes. J. Evol. Biol. 10:233-252

71. Lokki J, Saura A. 1980. Polyploidy in insect evolution. See Ref. 69, pp. 277312

72. Lopez Pajares I, Delicado A, Diaz de Bustamante A, Pellicer A, Pinel I, et al. 1990. Tetraploidy in a liveborn infant. J. Med. Genet. 27:782-83

73. Löve A, Löve D, Pichi Sermolli REG. 1977. Cytotaxonomical Atlas of the Pteridophyta. Vaduz, Germany: Cramer

73a. Lowcock LA. 1994. Biotype, genomotype, and genotype: variable effects of polyploidy and hybridity on ecological partitioning in a bisexual-unisexual 
community of salamanders. Can. J. Zool. 72:104-17

74. Lynch M, Force A. 2000. The probability of duplicate gene preservation by subfunctionalization. Genetics 154:459-73

75. Mabberley DJ. 1997. The Plant-Book. Cambridge: Cambridge Univ. Press

76. Mahony MJ, Robinson ES. 1980. Polyploidy in the Australian leptodactylid frog genus Neobatrachus. Chromosoma 81:199-212

77. Martin AP. 1999. Increasing genomic complexity by gene duplication and the origin of vertebrates. Am. Nat. 154:111-24

78. Martins MJ, Collares-Pereira MJ, Cowx IG, Coelho MM. 1998. Diploids v. triploids of Rutilus alburnoides: spatial segregation and morphological differences. J. Fish Biol. 52:817-28

79. Masterson J. 1994. Stomatal size in fossil plants: evidence for polyploidy in majority of angiosperms. Science 264:421-23

80. McFadden DE, Kwong LC, Yam IYL, Langlois S. 1993. Parental origin of triploidy in human fetuses: evidence for genomic imprinting. Hum. Genet. 1993:465-69

81. McLister JD, Stevens ED, Bogart JP. 1995. Comparative contractile dynamics of calling and locomotor muscles in three hylid frogs. J. Exp. Biol. 198:1527-38

82. Moore G, Devos KM, Wang Z, Gale MD. 1995. Cereal genome evolution. Grasses, line up and form a circle. Curr. Biol. 5:73739

83. Müller HJ. 1925. Why polyploidy is rarer in animals than in plants. Am. Nat. 59:34653

84. Nadeau JH, Sankoff D. 1997. Comparable rates of gene loss and functional divergence after genome duplications early in vertebrate evolution. Genetics 147:125966

85. Ness BD, Soltis DE, Soltis PS. 1990. An examination of polyploidy and putative introgression in Calochortus subsection Nudi (Liliaceae). Am. J. Bot. 77:1519-31

86. Nogler GA. 1984. Genetics of apospory in Ranunculus auricomus. V. Conclusions. Bot. Helvet. 94:411-22

87. Ohno S. 1970. Evolution by Gene Duplication. New York: Springer-Verlag

88. Orr HA. 1990. "Why polyploidy is rare in animals than in plants" revisited. Am. Nat. 136:759-70

89. Orr HA, Otto SP. 1994. Does diploidy increase the rate of adaptation? Genetics 136:1475-80

90. Otto SP, Barton NH. 1997. The evolution of recombination: removing the limits to natural selection. Genetics 147:879906

91. Otto SP, Goldstein DB. 1992. Recombination and the evolution of diploidy. Genetics 131:745-51

92. Otto SP, Whitlock MC. 1997. The probability of fixation in populations of changing size. Genetics 146:723-33

93. Paquin CE, Adams J. 1983. Frequency of fixation of adaptive mutations is higher in evolving diploid than haploid yeast populations. Nature 302:495-500

94. Pendleton JW, Nagai BK, Murtha MT, Ruddle FH. 1993. Expansion of the Hox gene family and the evolution of chordates. Proc. Natl. Acad. Sci. USA 90:6300-4

95. Perrot V, Richerd S, Valero M. 1991. Transition from haploidy to diploidy. Nature 351:315-17

96. Pessino SC, Ortiz JPA, Hayward MD, Quarin CL. 1999. The molecular genetics of gametophytic apomixis. Hereditas 130:1-11

97. Petit C, Thompson JD. 1999. Species diversity and ecological range in relation to ploidy level in the flora of the Pyrenees. Evol. Ecol. 13:45-66

98. Postlethwait JH, Yan YL, Gates MA, Horne S, Amores A, et al. 1998. Vertebrate genome evolution and the zebrafish gene map. Nat. Genet. 18:345-49

99. Ramsey J, Schemske DW. 1998. Pathways, mechanisms, and rates of polyploid formation in flowering plants. Annu. Rev. Ecol. Syst. 29:467-501 
100. Renner SS, Ricklefs RE. 1995. Dioecy and its correlates in the flowering plants. Am. J. Bot. 82:596-606

101. Roca JR, Ribas M, Baguna J. 1992. Distribution, ecology, mode of reproduction and karyology of freshwater planarians (Platyhelminthes; Turbellaria; Tricladida) in the springs of the central Pyrenees. Ecography 15:373-84

102. Scarbrough PR, Hersh J, Kukolich MK, Carroll AJ, Finley SC, et al. 1984. Tetraploidy: a report of three live-born infants. Am. J. Med. Genet. 19:29-37

103. Schmid M, Enderle E, Schindler D, Schempp W. 1989. Chromosome banding and DNA replication patterns in bird karyotypes. Cytogenet. Cell. Genet. 52:13946

104. Schultz RJ. 1980. Role of polyploidy in the evolution of fishes. See Ref. 69, pp. 313-40

105. Seoighe C, Wolfe KH. 1999. Yeast genome evolution in the post-genome era. Curr. Opin. Microbiol. 2:548-54

106. Sexton OJ. 1980. Polyploidy in animal evolution: summary. See Ref. 69, pp. 379-81

107. Shiono H, Azumi J, Fujiwara M, Yamazaki H, Kikuchi K. 1988. Tetraploidy in a 15-month-old girl. Am. J. Med. Genet. 29:543-47

108. Simmons MJ, Crow JF. 1977. Mutations affecting fitness in Drosophila populations. Annu. Rev. Genet. 11:49-78

109. Skrabanek L, Wolfe KH. 1998. Eukaryote genome duplication - where's the evidence? Curr. Opin. Genet. Dev. 8:694700

110. Soltis DE, Soltis PS. 1993. Molecular data and the dynamic nature of polyploidy. Crit. Rev. Plant Sci. 12:243-73

111. Soltis DE, Soltis PS. 1999. Polyploidy: recurrent formation and genome evolution. Trends Ecol. Evol. 14:348-52

112. Song KM, Lu P, Tang KL, Osborn TC. 1995. Rapid genome change in synthetic polyploids of Brassica and its implica- tions for polyploid evolution. Proc. Natl. Acad. Sci. USA 92:7719-23

113. Stebbins GL. 1938. Cytological characteristics associated with the different growth habits in the dicotyledons. Am. J. Bot. 25:189-98

114. Stebbins GL. 1950. Variation and Evolution in Plants. New York: Columbia Univ. Press

115. Stebbins GL. 1971. Chromosomal Evolution in Higher Plants. London: Edward Arnold

116. Stebbins GL. 1971. Processes of Organic Evolution. Englewood Cliffs, NJ: Prentice-Hall

117. Stebbins GL. 1980. Polyploidy in plants: unsolved problems and prospects. See Ref. 69, pp. 495-520

118. Thompson JD, Lumaret R. 1992. The evolutionary dynamics of polyploid plants: origins, establishment and persistence. Trends Ecol. Evol. 7:302-7

119. Uchida IA, Freeman VC. 1985. Triploidy and chromosomes. Am. J. Obst. Gynecol. 151:65-69

120. Uhl CH. 1978. Chromosomes of Mexican Sedum, II Section Pachysedum. Rhodora 80:491-512

121. Uyeno T, Smith GR. 1972. Tetraploid origin of the karyotype of catostomid fishes. Science 175:644-46

122. Viktorov AG. 1997. Diversity of polyploid races in the family Lumbricidae. Soil Biol. Biochem. 29:217-21

123. Villar R, Veneklaas EJ, Jordano P, Lambers H. 1998. Relative growth rate and biomass allocation in 20 Aegilops (Poaceae) species. New Phytol. 140:42537

124. Vogel G. 1998. Doubled genes may explain fish diversity. Science 281:1119-21

125. von Well E, Fossey A. 1998. A comparative investigation of seed germination, metabolism and seedling growth between two polyploid Triticum species. Euphytica 101:83-89

126. von Wettstein F. 1927. Die Erscheinung 
der Heteroploidie, besonders im Pflanzenreich. Ergeb. Biol. 2:311-56

127. Wagner WH, Wagner FS. 1980. Polyploidy in pteridophytes. See Ref. 69, pp. 199-214

128. Walsh JB. 1995. How often do duplicated genes evolve new functions? $\mathrm{Ge}$ netics 139:421-28

128a. Wedderburn FM, Richards AJ. 1992. Secondary homostyly in Primula L.: evidence for the model of the "S" supergene. New Phytol. 121:649-55

129. Weiss RL, Kukora JR, Adams J. 1975. The relationship between enzyme activity, cell geometry, and fitness in Saccha- romyces cerevisiae. Proc. Nat. Acad. Sci. USA 72(3):794-98

129a. Wendel JF. 2000. Genome evolution in polyploids. Plant Mol. Biol. 42:225-49

130. White MJD. 1973. Animal Cytology and Evolution. Cambridge: Univ. Cambridge Press. 3rd ed.

131. Wolfe KH, Shields DC. 1997. Molecular evidence for an ancient duplication of the entire yeast genome. Nature 387:708-13

132. Wright S. 1969. Evolution and Genetics of Populations. II. The Theory of Gene Frequencies. Chicago: Univ. Chicago Press 